Published in final edited form as:

ACS Appl Mater Interfaces. 2018 February 07; 10(5): 4379-4393. doi:10.1021/acsami.7b14362.

\title{
Melanocortin-1 Receptor-Targeting Ultrasmall Silica Nanoparticles for Dual-Modality Human Melanoma Imaging
}

\author{
Feng Chen ${ }^{1, \dagger}$, Xiuli Zhang ${ }^{2, \dagger}$, Kai Ma ${ }^{3, \dagger}$, Brian Madajewski ${ }^{1}$, Miriam Benezra ${ }^{1}$, Li Zhang ${ }^{1}$, \\ Evan Phillips $^{1,+}$, Melik Z. Turker ${ }^{3}$, Fabio Gallazzi ${ }^{2}$, Michael Overholtzer ${ }^{4,5}$, Mohan Pauliah ${ }^{1}$, \\ Mithat Gonen ${ }^{6}$, Pat Zanzonico ${ }^{7}$, Ulrich Wiesner ${ }^{3,{ }^{*}}$, Michelle S. Bradbury ${ }^{1,{ }^{*}}$, and Thomas P. \\ Quinn', ${ }^{*}$ \\ ${ }^{1}$ Department of Radiology, Sloan Kettering Institute for Cancer Research, New York, NY 10065, \\ USA
}

${ }^{2}$ Department of Biochemistry, University of Missouri, Columbia, MO 65211, USA and Harry S Truman Veterans' Hospital, Columbia, MO 65201 USA

${ }^{3}$ Department of Materials Science \& Engineering, Cornell University, Ithaca, NY 14853, USA

${ }^{4}$ Cell Biology Program, Sloan Kettering Institute for Cancer Research, New York, NY 10065, USA

${ }^{5}$ BCMB Allied Program, Weill Cornell Medical College, New York, NY 10065, USA

${ }^{6}$ Department of Epidemiology and Biostatistics, Sloan Kettering Institute for Cancer Research, New York, NY 10065, USA

${ }^{7}$ Department of Medical Physics, Sloan Kettering Institute for Cancer Research, New York, NY 10065, USA

${ }^{8}$ Molecular Pharmacology Program, Sloan Kettering Institute for Cancer Research, New York, NY 10065, USA

Correspondence and requests for materials should be addressed to: T. Quinn, quinnt@ missouri.edu, M.S. Bradbury,

bradburm@mskcc.org, and U.Wiesner, ubw1@ cornell.edu.

†FC, XZ, KM contributed equally to the work.

*UW, MB, TQ contributed equally to the work.

+Present address: Weldon School of Biomedical Engineering, Purdue University, West Lafayette, Indiana 47907 USA

Present Addresses

Department of Biochemistry, University of Missouri, Columbia, MO 65211, USA and Harry S Truman Veterans' Hospital, Columbia, MO 65201 USA

Department of Radiology and Molecular Pharmacology Program, Sloan Kettering Institute for Cancer Research, New York, New York 10065, USA.

Department of Materials Science \& Engineering, Cornell University, Ithaca, New York 14853, USA.

Author Contributions

The manuscript was written through contributions of all authors. All authors have given approval to the final version of the manuscript. ASSOCIATED CONTENT

Supporting Information. This material is available free of charge via the Internet at http://pubs.acs.org. Sequences of four custom-

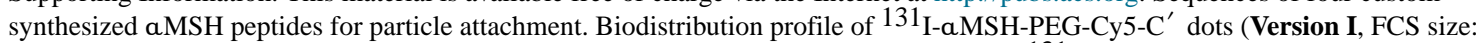

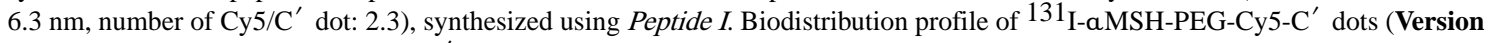
II, FCS size: $4.8 \mathrm{~nm}$, number of Cy5/C' dot: 2.6), synthesized using Peptide II. Viability and proliferation of M21 cells and B16F10 cells as a function of particle concentration and incubation time. Deconvolution of the UV-Vis spectrum of aMSH from the spectrum of aMSH-PEG-Cy5-C ${ }^{\prime}$ dots. PET/CT maximum intensity projection (MIP) images at 2 hours after i.v.-injection of ${ }^{124}$ I-aMSH-PEG-

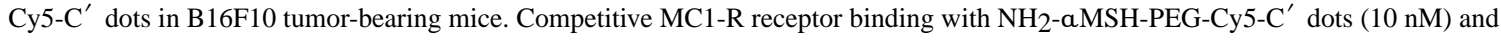
NDP blocking peptide (100 mg, or $50 \mathrm{mg} / \mathrm{mL}$ ) in B16F10 cells. Tables of biodistribution data from M21 (and B16F10) tumor-bearing mice injected with ${ }^{125}$ I-aMSH-PEG-Cy5-C ${ }^{\prime}$ dots. Tables of tumor-to-background ratios (blood, muscle, and liver) from M21 (and B16F10) tumor-bearing mice injected with ${ }^{125}$ I-aMSH-PEG-Cy5- $\mathrm{C}^{\prime}$ dots. 


\section{Abstract}

The poor prognosis associated with malignant melanoma has not changed substantially over the past 30 years. Targeted molecular therapies, such as immunotherapy have shown promise, but suffer from resistance and off-target toxicities, underscoring the need for alternative therapeutic strategies that can be used in combination with existing protocols. Moreover, peptides targeting melanoma-specific markers, like the melanocortin-1 receptor (MC1-R), for imaging and therapy exhibit high renal uptake that limits clinical translation. In the current study, the application of ultrasmall fluorescent (Cy5) silica nanoparticles ( $\mathrm{C}^{\prime}$ dots), conjugated with $\mathrm{MC} 1-\mathrm{R}$ targeting alpha melanocyte stimulating hormone (aMSH) peptides on the polyethylene glycol (PEG) coated surface, is examined for melanoma-selective imaging. aMSH peptide sequences, evaluated for conjugation to the PEG-Cy5- $\mathrm{C}^{\prime}$ dot nanoparticles, bound to MC1-R with high affinity, and targeted melanoma in syngenetic and xenografted melanoma mouse models. Results demonstrated a 10-fold improvement in MC1-R affinity over the native peptide alone following surface attachment of the optimal aMSH peptide. Systematic in vivo studies further demonstrated favorable in vivo renal clearance kinetics as well as receptor-mediated tumor cell internalization of as-developed radiolabeled particle tracers in $\mathrm{B} 16 \mathrm{~F} 10$ melanoma bearing mice. These findings highlight the ability of aMSH-PEG-Cy5- $\mathrm{C}^{\prime}$ dots to overcome previous hurdles that prevented clinical translation of peptide and antibody-based melanoma probes, and reveal the potential of aMSH-PEG-Cy5-C $\mathrm{C}^{\prime}$ dots for melanoma selective imaging, image-guided surgery, and therapeutic applications.

\section{Graphical Abstract}
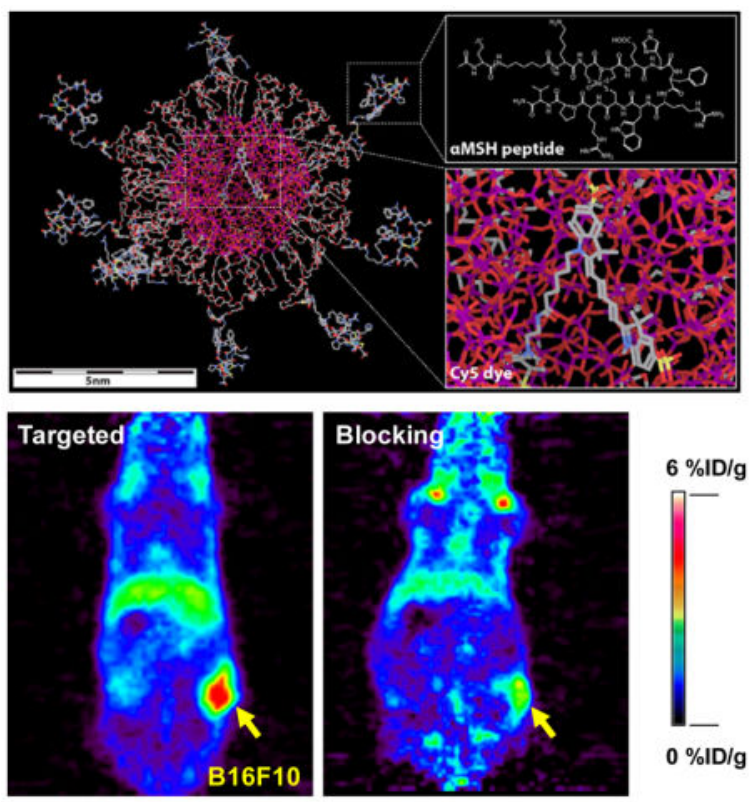

\section{Keywords}

aMSH peptide; Ultrasmall silica nanoparticle; $C^{\prime}$ dots; Target-or-clear; Melanoma 


\section{INTRODUCTION}

Melanoma is the deadliest form of skin cancer. Although early diagnosis and treatment results in a high percentage of cures, the survival rate significantly declines to only 15-20\% for systemic melanoma disease spread. ${ }^{1}$ The mortality rate from melanoma has not changed substantially over the past 25 years. In addition, mechanisms underlying melanoma treatment resistance remain elusive. Treatment resistance is likely due to a combination of immune surveillance avoidance, multidrug resistance, and persistent melanoma stem cell populations. ${ }^{2-4}$ However, therapeutic exploitation of genetic mutations in melanoma and the discovery of targets for cancer immunotherapies have resulted in better treatment paradigms for patients. ${ }^{5-10}$ As a result of advancing such novel targeted treatment options, improved clinical responses have been achieved, although generally short-lived (6-12 months) and accompanied by immune resistance mechanisms and/or activation of alternative signaling pathways. ${ }^{2,5,11}$ Of critical need, therefore, are alternative systemic therapeutic options that target new melanoma-specific signaling pathway markers.

The melanoma biomarker, melanocortin-1 receptor (MC1-R), is overexpressed on the surface of human melanoma tumor cells, and serves as an attractive target for melanomaspecific imaging and the selective delivery of therapeutic agents. ${ }^{12-18}$ Its naturally occurring ligand is a 13-amino acid peptide, alpha melanocyte stimulating hormone (aMSH), which is involved in the regulation of skin pigmentation, as well as immune and anti-inflammatory responses. ${ }^{19}$ Numerous structure-activity studies have identified linear and cyclic peptide agonists and antagonists with nanomolar and subnanomolar binding affinities. ${ }^{20}$ Binding of MC1-R agonists to receptors expressed on melanoma cells results in rapid cellular internalization of the receptor-peptide complex, and this mechanism has been exploited for receptor-mediated internalization of radiolabeled, optical, and therapeutic peptide complexes.

Potent radiolabeled peptide analogs of the aMSH sequence, such as [Nle $\left.{ }^{4}, \mathrm{D}-\mathrm{Phe}^{7}\right]-\mathrm{aMSH}$ (NDP) ${ }^{21}$ have been extensively studied as imaging and therapeutic agents. Although early NDP radioconjugates ${ }^{22,23}$ suffered from low tumor retention and poor biodistribution properties, cyclization of the core MC1-R binding region of the aMSH sequence via metal coordination or lactam bond formation resulted in radiolabeled peptides with high tumor uptake and prolonged retention. ${ }^{16,24-25}$ However, their high non-specific uptake in radiosensitive organs, such as the kidneys, together with limited mid-gut imaging sensitivity and low tumor-to-background ratios, have hampered clinical translation efforts. ${ }^{15,26}$

To overcome these limitations, several strategies have been advanced, including linker optimization, peptide multimerization, and the attachment of peptides onto nanoparticle surfaces. Optimization of linkers bridging the peptide with a radiometal chelator have led to incremental improvements in tumor-to-kidney ratios, while the greatest improvements were found to occur after altering the net charge of the entire molecular complex. ${ }^{15-16}$ Further, the multimerization of aMSH peptides has resulted in constructs with improved in vitro cell binding affinities. However, these same constructs demonstrated poor in vivo pharmacokinetic properties due to increased hydrophobicity and reduced tissue penetration.

${ }^{27}$ Finally, the use of different types of nanomaterials, such as liposomes and dendrimers, 
conjugated with multiple copies of aMSH analogs, has generally resulted in only modest increases in in vitro $\mathrm{MC1}-\mathrm{R}$ binding affinities. ${ }^{28}$ Although preliminary in vivo MC1-R targeting studies utilizing larger diameter aMSH-conjugated particles, such as polymer micelles ${ }^{29}$ (i.e., $\sim 100 \mathrm{~nm}$ ) and hollow gold nanospheres ${ }^{30}$ (i.e., $40 \mathrm{~nm}$ ) have been reported, high non-specific accumulation of these products in the reticuloendothelial system (RES) has led to significantly decreased target-to-background ratios ${ }^{31}$ and/or raised concerns for off-target toxicity, thereby hindering their translational potential. As a result, successful translation of an MC1-R targeted imaging platform demonstrating favorable biological and clearance properties has not been achieved.

Herein we report on the structure-activity relationships for a small library of customsynthesized rhenium (Re)-cyclized aMSH peptide ligands linked to positron emission tomography (PET) imaging labels conjugated to latest-generation ultrasmall "target-orclear" silica nanoparticles (i.e., Cornell prime dots or $\mathrm{C}^{\prime}$ dots) with different surface chemical designs. ${ }^{32-33}$ These efforts are based on earlier work in which $\mathrm{C}^{\prime}$ dots were surface engineered with controllable numbers of surface functional groups. ${ }^{34-36} \mathrm{We}$ characterize the resulting ultrasmall (diameter $<10 \mathrm{~nm}$ ) MC1-R targeting and dual-modality $\mathrm{C}^{\prime}$ dot conjugates (Scheme 1) in terms of their photophysical and in vitrolin vivo biological properties in melanoma cell lines and xenograft tumor models in comparison to the native aMSH peptide and/or particle and biological controls. The coupling of different radiolabels, such as radiohalogens (i.e., iodine- $125 ;{ }^{125} \mathrm{I}$ ) or radiometals (i.e., zirconium-89; ${ }^{89} \mathrm{Zr}$ ), to the particle surface facilitates comparisons of the pharmacokinetics (PK), clearance, and/or tumor-targeting profiles of these radioconjugates. Results demonstrated statistically significant increases in MC1-R-specific tumor-targeted uptake and target-to-background ratios of ${ }^{89} \mathrm{Zr}$-DFO-aMSH-PEG-Cy5-C' dots over the time course of the study, as well as internalization of the targeted particle probes in tumor tissue specimens. No significant difference was found in the PK profiles measured at 24 hours post-injection (p.i.) for particles labeled with different radionuclides. We hope these findings will serve as the foundation for next-stage diagnostic and/or radiotherapeutic developments by exploiting a variety of radiometals for the image-guided treatment of human melanoma.

\section{RESULTS AND DISCUSSION}

\section{Synthesis and screening of MC1-R targeting peptide(s)}

MC1-R targeting peptide ligands used for $\mathrm{C}^{\prime}$ dot synthesis were developed on the basis of the ReCCMSH molecule (Ac-Re[Cys ${ }^{3}-\mathrm{Cys}^{4}$-Glu ${ }^{5}$-His ${ }^{6}$-d-Phe ${ }^{7}-\mathrm{Arg}^{8}{ }^{8}$ Trp $^{9}$-Cys $\left.{ }^{10}\right]$-Arg ${ }^{11}$ Pro ${ }^{12}-\mathrm{Val}^{13}-\mathrm{NH}_{2}$; superscripts refer to amino acid number based on the native aMSH sequence), and cyclized via rhenium (Re) coordination by Cys-3, Cys-4, and Cys-10 sidechain thiols as well as the Cys-4 amide. ${ }^{24,37}$ The initial strategy involved modification of the de-acetylated ReCCMSH molecule (Figure S1a; Peptide I) with N-succinimidyl Sacetylthiolacetate (SATA) and the Bolton-Hunter reagent, N-succinimidyl-3-(4hydroxypheynyl) propionate (SHPP). The SATA reaction and deprotection converted a primary amine to a free thiol for thiol-ene click chemistry with a heterobifunctional maleimide containing PEG used in the $\mathrm{C}^{\prime}$ dot synthesis, while SHPP conjugation enabled the ReCCMSH-C ${ }^{\prime}$ dot to be radioiodinated. Radiochemical yields of the ${ }^{131}$ I-ReCCMSH- 
PEG-C'dots were poor $(<2 \%)$, and an initial biodistribution study demonstrated transient tumor uptake and GI clearance (Figure S2a). A second ReCCMSH peptide analog was synthesized with a pendant tyrosine residue for direct radioiodination (Figure S1b: Peptide II). The amino terminus of the peptide was modified by SATA to facilitate $\mathrm{C}^{\prime}$ dot synthesis. The radiolabeling yield for the Ser-Tyr $\left({ }^{131} \mathrm{I}\right)$-Ser-ReCCMSH-PEG-Cy5-C ${ }^{\prime}$ dots was $\sim 7 \%$. Preliminary biodistribution results showed poor tumor uptake and evidence of in vivo deiodination (Figure S2b). The third peptide design (Figure S1c: Peptide III) contained a double aminohexanoic acid $(\mathrm{Ahx})_{2}$-linker between an N-terminal Ac-Cys and the D-LysReCCMSH peptide (Figure 1a). The D-Lys sidechain could be modified for radiolabeling via the addition of prosthetic groups (i.e., SHPP) or metal chelators. Ac-Cys-(Ahx) $)_{2}$-D-Lys$\mathrm{Re}\left[\right.$ Cys-Cys-Glu-His-dPhe-Arg-Trp-Cys]-Arg-Pro-Val-NH $\mathrm{N}_{2}$ (aMSH, Figure S1c) was conjugated with maleimide-terminated heterobifunctional PEG-silane molecules via the $\mathrm{N}$ terminal Cys thiol in preparation for nanoparticle PEGylation. The aMSH peptide was synthesized in its entirety using Fmoc chemistry, and high-purity batches could be produced at a large scale (Figure 1b). The $\mathrm{IC}_{50}$ value measured for the MC1-R targeting D-LysReCCMSH peptide was $6.2 \times 10^{-9} \mathrm{M}$ (Figure 1c). A scrambled aMSH peptide (Scr-MSH: Ac-Cys-(Ahx) $)_{2}$-D-Lys-Re[Cys-Cys-Trp-Arg-His-dPhe-Glu-Cys]-Arg-Pro-Val-NH ${ }_{2}$, Figure S1d) was also designed as a negative control for MC1-R binding. The $\mathrm{IC}_{50}$ of the scrambled peptide was $3.7 \times 10^{-6} \mathrm{M}$ (data not shown). Peptide III was found to be superior to Peptides I and II in terms of synthetic yield and/or stability, and therefore was selected as the lead candidate for aMSH-PEG-C ${ }^{\prime}$ dot synthesis and characterization and for performing in vitro and in vivo MC1-R targeting studies.

\section{Synthesis and characterization of aMSH -PEG-Cy5-C' dots}

The aMSH-PEG-Cy5-C $\mathrm{C}^{\prime}$ dots described in this paper were prepared using the latest synthesis protocol of Cornell dots (C dots). ${ }^{32}$ This new generation of Cornell dots is referred to as Cornell prime dots, or in short $\mathrm{C}^{\prime}$ dots. As compared to the original conventional Stöber process derived $\mathrm{C}$ dots using ethanol as the reaction medium, ${ }^{38-39}$ the synthesis of $\mathrm{C}^{\prime}$ dots is now conducted in water. Fast hydrolysis and condensation of silane molecules in an aqueous environment facilitates the formation of homogeneous ultra-small silica nanoparticles, ${ }^{32,40-41}$ which is different from the process associated with larger size (>10 nm HD) silica particles. ${ }^{42}$ At the same time, silane-modified Cy5 dyes were added together with the silica source to be covalently encapsulated in the silica nanoparticles during their formation, endowing the particles with bright near-infrared fluorescence. ${ }^{43-45}$ Additionally, the aqueous environment also promotes the formation of hydrogen bonds between silica surface and peptide ligands. ${ }^{33}$ As a result, by adding monofunctional PEG-silane and aMSH modified heterobifunctional aMSH-PEG-silane into the reaction mixture, PEG-silane and and in particular aMSH-PEG-silane were quickly adsorbed by the silica nanoparticle surface, terminating the particle growth. ${ }^{33,41}$ An overnight high temperature treatment at $80{ }^{\circ} \mathrm{C}$ was then applied to further enhance the covalent attachment of PEG-silane and aMSH-PEG-silane to the nanoparticles. The resulting aMSH-PEG-Cy5- $\mathrm{C}^{\prime}$ dots were finally purified by gel permeation chromatography (GPC), ${ }^{34,36}$ and characterized by a combination of different techniques, including GPC, transmission electron microscopy (TEM), ultraviolet-visible (UV-Vis) spectroscopy, and fluorescence correlation spectroscopy (FCS). 
The aMSH-PEG-Cy5-C ${ }^{\prime}$ dots synthesized through this approach (Scheme 1) contained Cy5 fluorescent dyes in the silica matrix. The particle surface was modified with PEG chains, ensuring their high bio-compatibility. aMSH peptides were covalently attached at the end of some of the PEG chains, enabling active tumor targeting. The GPC elugram of the resulting purified aMSH-PEG-Cy5- $\mathrm{C}^{\prime}$ dots showed only a single peak at around 9 min, which was well fitted by a single, symmetric Gaussian distribution function (Figure 2a), demonstrating high purity of the final particle product and narrow particle size distribution, consistent with TEM observations (inset of Figure 2a). ${ }^{34,36}$ The average hydrodynamic diameter of the aMSH-PEG-Cy5-C $\mathrm{C}^{\prime}$ dots was $6.2 \mathrm{~nm}$, according to a fit of the fluorescence correlation spectroscopy (FCS) correlation curve (Figure $2 b$ ). In addition to particle size, the FCS fit also provides particle concentration, which in combination with optical spectroscopy can be used to estimate particle fluorescence brightness and average numbers of functional ligands on each particle. ${ }^{36,43}$ The UV-Vis spectrum of the aMSH-PEG-Cy5-C' ${ }^{\prime}$ dots exhibited an absorbance peak at around 650nm, consistent with the absorbance of Cy5 dye. In addition, strong absorbance in the regime of wavelength $<300 \mathrm{~nm}$ was observed, which was attributed to the aMSH peptides attached to the $\mathrm{C}^{\prime}$ dot surface (Figure 2c). As demonstrated for other ligands before, the UV-Vis spectrum of aMSH-PEG-Cy5-C' dots was deconvoluted into the contributions from Cy5-PEG-Cy5-C' dots and aMSH, through fitting the spectrum by a set of standard spectra of individual components (Figure 2d, e; Figure S4). ${ }^{36}$ Through dividing the ligand concentrations obtained from UV-Vis spectral analysis by the particle concentration obtained from FCS, the average numbers of Cy5 dyes and aMSH peptides per particle were estimated to be 1.5 and 6.9 , respectively.

\section{In vitro receptor targeting and cellular uptake studies of aMSH-PEG-Cy5-C' dots}

The in vitro cell binding kinetics and targeting specificity of aMSH-PEG-Cy5- $\mathrm{C}^{\prime}$ dots bearing, on average, about $7 \mathrm{aMSH}$ ligands per $\mathrm{C}^{\prime}$ dot, were evaluated by flow cytometry in murine and human MC1-R-overexpressing cell lines, B16F10 and M21, respectively, using MC1-R receptor blocking assays and particle controls (i.e., Scr-MSH-PEG-Cy5-C ${ }^{\prime}$ dots). Figure 3a shows the concentration-dependent cell binding and uptake of aMSH-PEG-Cy5-C ' dots in B16F10 and M21 cells, as against that found in media alone (0.5\% BSA). Enhanced particle uptake in both cell lines was observed with increasing particle concentrations (i.e., $0-250 \mathrm{nM}$ ) over a 4 hour incubation period. Saturation binding was seen to occur at $\sim 100 \mathrm{nM}$ for the B16F10 cell line and $150 \mathrm{nM}$ for M21 cells, which is likely related to known differences in the regulation of MC1-R expression in mouse and human cells, ${ }^{46}$ a combination of receptor density, ${ }^{14,47}$ internalization kinetics, and receptor recycling rates. Furthermore, a MSH-PEG-C' ${ }^{\prime}$ dot uptake in B16F10 and M21 cells using $100 \mathrm{nM}$ concentrations was seen to increase as a function of incubation time by flow cytometry (Figure 3b), noting that maximum values for both cell types were found at 4-5 hours.

To assess in vitro MC1-R binding specificity, blocking studies using an anti-MC1-R antibody were performed by using flow cytometry. As shown in Figure 3, competitive binding assays showed that uptake via MC1-R was almost completely blocked ( 90\%) in M21 cells (Figure 3c) and partially blocked (i.e., > 60\%) in B16F10 cells (Figure 3d) using excess (×6-9) anti-MC1-R antibody prior to addition of $25 \mathrm{nM}$ aMSH-PEG-Cy5-C' dots. 
Differences in the degree of receptor blocking may be attributed to variations in the rates of MC1-R internalization and/or recycling rates to the cell surface in mouse and human melanoma cells. For instance, endocytosed MC1-R receptors in B16 cells do not recycle back to the cell surface, ${ }^{46}$ so that fewer receptors may be available for binding. Targeting specificity of aMSH-PEG-Cy5-C' dots was further demonstrated in B16F10 cells by MC1$\mathrm{R}$ receptor binding with $25 \mathrm{nM}$ of Scr-MSH-PEG-Cy5- $\mathrm{C}^{\prime}$ dots under the same incubation conditions, and findings were similar to those found with anti-MC1-R antibody (i.e., $>60 \%$; Figure 3e).

These results were confirmed by competitive binding assays, which measured the $\mathrm{IC}_{50}$ of aMSH-PEG-Cy5-C' dots for MC1-R using B16F10 melanoma cells and a radioiodinated aMSH analog ${ }^{125} \mathrm{I}-\left(\mathrm{Tyr}^{2}\right)-\left[\mathrm{Nle}^{4}, \mathrm{D}-\mathrm{Phe}^{7}\right]-\mathrm{aMSH}\left({ }^{125} \mathrm{I}-\mathrm{NDP}\right)$, the latter exhibiting picomolar affinity for MC1-R. ${ }^{21}$ The $\mathrm{IC}_{50}$, or concentration of nanoparticle required to inhibit $50 \%$ of a standard radiolabeled MC1-R agonist binding, was determined for both aMSH-PEG-C ${ }^{\prime}$ dot and Scr-MSH-PEG-C ${ }^{\prime}$ dot constructs over concentrations ranging from $10^{-13}$ to $10^{-5} \mathrm{~mol} / \mathrm{L}$ (Figure 3f). The $\mathrm{IC}_{50}$ value for aMSH-PEG-Cy5-C dots was estimated to be $6.6 \times 10^{-10} \mathrm{M}$, while that for the negative particle control (i.e., Scr-MSH-PEG-Cy5-C' dots) was $2.3 \times 10^{-8}$ $\mathrm{M}$, suggesting that differences in MC1-R affinity between the aMSH-PEG-Cy5-C ${ }^{\prime}$ dots and the Scr-MSH-PEG-Cy5- $\mathrm{C}^{\prime}$ dots were more than two orders of magnitude.

Intracellular localization of aMSH-functionalized $\mathrm{C}^{\prime}$ dots in M21 and B16F10 cells was found to occur primarily via an MC1-R-driven endocytic mechanism, similar to that found for MC1-R targeting ligand-receptor complexes. ${ }^{48}$ To assess whether this process was temperature-dependent, and whether attachment of aMSH peptides to the surfaces of such ultrasmall particles promoted their accumulation in M21 and B16F10 cells, the uptake of particle-exposed cells was investigated at 3 different temperatures (i.e., $4^{\circ}, 25^{\circ} \mathrm{C}$ and $37^{\circ} \mathrm{C}$ ) over a 4-hour incubation period. The results, summarized in Figures $4 \mathrm{a}$ and $4 \mathrm{~b}$, demonstrated that cellular internalization of aMSH-PEG-Cy5- $\mathrm{C}^{\prime}$ dots progressively rose in both M21 (Figure 4a) and B16F10 (Figure 4b) cells as a function of incubation temperatures. In addition, significantly decreased particle uptake was observed at $4^{\circ} \mathrm{C}$ (i.e., $>95 \%$ ) relative to that found at $37^{\circ} \mathrm{C}$, suggesting that intracellular internalization occurred primarily via MC1-R-dependent receptor-mediated endocytosis. ${ }^{49-50}$ Similar results were observed for early-generation cRGDY-functionalized $C$ dots in $\alpha_{\nu} \beta_{3}$ integrin-overexpressing cell lines. ${ }^{51}$ Representative microscopic imaging of M21 cells (Figure 4c) 4 hours after particle exposure showed that internalized aMSH-PEG-Cy5- $\mathrm{C}^{\prime}$ dots resided in late endosomes/lysosomes, as demonstrated by co-localization with an expressed LAMP1-GFP protein that labels these compartments.

To demonstrate that no significant loss of cell viability or proliferative activity occurred due to the presence of particles, both M21 and B16F10 cells were exposed to aMSH-PEG-Cy5$\mathrm{C}^{\prime}$ dots over a wide range of concentrations $(0-250 \mathrm{nM})$ and incubation times $(0-96 \mathrm{hrs})$ in serum-supplemented media at $37^{\circ} \mathrm{C}$. In both cases, no significant loss of cell survival was observed at two post-incubation times relative to findings seen under control conditions (Figure S3a, 3b). Furthermore, time-dependent increases in the number of cells were found following multi-dose $(\mathrm{n}=4)$ addition of $100 \mathrm{nM}$ particles to M21 and B16F10 cells, suggesting no adverse effects on cellular proliferation (Figure S3c). Taken together, these 
results demonstrate site-specific targeting, cellular internalization via endolysosomal pathways, and an absence of cytotoxicity of aMSH-PEG-Cy5-C' dots in MC1-Roverexpressing cell lines.

\section{Biodistribution and radiodosimetry of ${ }^{125}$ I-aMSH-PEG-Cy5-C' dots}

After demonstrating MC1-R specific cellular targeting in vitro, we studied the PK of radiolabeled aMSH-PEG-Cy5-C ${ }^{\prime}$ dots in MC1-R-overexpressing tumor models. Assynthesized aMSH-PEG-Cy5-C' $\mathrm{C}^{\prime}$ dots were initially radiolabeled with iodine-125 ( $\left.{ }^{125} \mathrm{I}\right)$ by reacting ${ }^{125}$ I-Bolton-Hunter reagent with primary amines associated with the aMSH-bound $\mathrm{C}^{\prime}$ dots, then purified using a PD-10 column to remove any free, unreacted ${ }^{125}$ ( see experimental section for more details). The in vivo biological properties of intravenously (i.v.)-injected ${ }^{125} \mathrm{I}$-aMSH-PEG-Cy5-C' ${ }^{\prime}$ dots $(\sim 50 \mu \mathrm{Ci}$, or $\sim 1.85 \mathrm{MBq})$, including their PK, tumor-targeting efficiency, and target-to-background ratios were then evaluated over a range of post-injection time points (i.e., 1, 4, 24, 72 and $96 \mathrm{~h}$ ) in both M21 and B16F10 xenografted mice, as shown in Figure 5. Favorable PK findings were observed for the particle tracer, including a reasonably long blood circulation half-time, dominant renal clearance, and minimal reticuloendothelial system (RES) uptake-results significantly different from those found for the native peptide radiotracer, specifically rapid renal clearance and high renal retention. ${ }^{15,26}$

As shown in Figure 5a and Table S1, whole-blood activity concentration of ${ }^{125} \mathrm{I}$-aMSHPEG-C ${ }^{\prime}$ dots, obtained from M21 tumor-bearing mice, was found to be $\sim 20 \% \mathrm{ID} / \mathrm{g}$ at early p.i. time points, gradually declining thereafter to $\sim 9$ and $\sim 1 \% \mathrm{ID} / \mathrm{g}$ at 24 and $96 \mathrm{~h}$ p.i., respectively. Dominant renal excretion was confirmed by the high activity measured in urine specimens, namely, $>100 \% \mathrm{ID} / \mathrm{g}$ at $1 \mathrm{~h} p . i$. and $>30 \% \mathrm{ID} / \mathrm{g}$ at $4 \mathrm{~h} p . i$. (Figure $5 \mathrm{~b}$ ). Renal uptake was only about $5 \% \mathrm{ID} / \mathrm{g}$ at 4 hours $p . i$., followed by a decline in the activity to $<1 \% \mathrm{ID} / \mathrm{g}$ at $96 \mathrm{~h} p . i$. Liver and spleen uptakes were also relatively low (i.e., $<5 \% \mathrm{ID} / \mathrm{g}$ at 24 h p.i. and $2 \% \mathrm{ID} / \mathrm{g}$ at $96 \mathrm{~h}$ p.i.). Maximal ${ }^{125} \mathrm{I}$-aMSH-PEG-Cy5-C' dot uptake in M21 tumors was estimated to be 5-6\%ID/g. Given the clearance of ${ }^{125}$ I-aMSH-PEG-Cy5-C ${ }^{\prime}$ dots from the circulation and tumor retention of the particles, tumor-to-blood ratios were observed to increase from $0.2 \pm 0.1$ at $1 \mathrm{~h} p . i$. to $5.8 \pm 2.7$ at $96 \mathrm{~h} p . i$. (Figure 5a-inset, Table S2). Maximal tumor-to-muscle and tumor-to-liver ratios were found to be $8.3 \pm 3.0$ and $1.8 \pm 0.6$, respectively (Figure 5a-inset, Table S2). A similar PK profile was found in mice bearing MC1-R-overexpressing B16F10 xenografts, namely increased blood and urinary activity concentrations at early p.i. time points, as well as low kidney and RES uptake (Figure 5c and d, Table S3 and S4). It is important to note that B16F10 tumor-bearing mice voided before urine specimens were obtained at $1 \mathrm{~h} \mathrm{p.i.,} \mathrm{thereby} \mathrm{leading} \mathrm{to} \mathrm{underestimates} \mathrm{of}$ the $\% \mathrm{ID} / \mathrm{g}$ for that time point (Figure $5 \mathrm{~d}$ ). While uptake of ${ }^{125} \mathrm{I}_{\text {-aMSH-PEG-C }}{ }^{\prime}$ dots in B16F10 xenografts was roughly equivalent to that found in M21 xenograft models at early time points, progressively reduced uptake was measured for B16F10 tumors (from $\sim 4.8 \% \mathrm{ID} / \mathrm{g}$ down to $1.2 \% \mathrm{ID} / \mathrm{g}$ ) at later p.i. time points (i.e., 72 and $96 \mathrm{~h}$ ) compared to that found for M21 tumors, presumably due to the rapid tumor doubling time (i.e., about 4 days) of the former. In both cases, over $90 \%$ of the injected ${ }^{125}{ }^{\text {I-aMSH-PEG-Cy5-C }}{ }^{\prime}$ dots were renally excreted within the first 4 days $p . i$. 
On the basis of appropriate organ mass-adjusted translation of the foregoing biodistribution data (Figure 5) to humans, time-integrated activities and human (70-kg Reference Person) normal-organ radiation absorbed dose were derived using the OLINDA computer program (yielding doses expressed in $m G y / M B q^{125}$ I administered). ${ }^{52}$ The PK profiles of ${ }^{125} \mathrm{I}$ aMSH-PEG-C' ${ }^{\prime}$ dots in both MC1-R-overexpressing tumor models and their normal-organ radiation doses (Table 1) were found to be nearly equivalent; the latter were also comparable to those of commonly used diagnostic radiotracers.

\section{In vivo tumor-targeted PET imaging of ${ }^{89}$ Zr-DFO-aMSH-PEG-Cy5-C' dots}

aMSH MSH-PEG-Cy5-C' dots in B16F10 xenografted mice, in vivo PET/CT imaging was initially performed after i.v.-injection of aMSH-PEG-Cy5- $\mathrm{C}^{\prime}$ dots conjugated with BoltonHunter reagent and radiolabeled with the positron-emission radioisotope, iodine-124 $\left({ }^{124} \mathrm{I}\right.$, $\mathrm{t}_{1 / 2}=4.2 \mathrm{~d}$ ). Enhanced tumor uptake was observed in this MC1-R-targeted cohort relative to that found in a second targeted cohort blocked with NDP. It should be noted that mice were not pretreated with KI to prevent free radioiodine uptake. Activity in the thyroid gland and stomach was felt to be a consequence of free iodine-124 associated with the specific radioiodination conditions needed to achieve high specific activity ${ }^{124}$ I-aMSH-PEG-C ${ }^{\prime}$ dot preparations, along with excess non-specifically bound iodine (Figure S5).

To address issues related to free iodine, zirconium-89 $\left({ }^{89} \mathrm{Zr}, \mathrm{t}_{1 / 2}=3.3 \mathrm{~d}\right)$ with a lower mean $\beta$ ${ }^{+}$energy ( $396 \mathrm{keV}$ vs. $820 \mathrm{keV}$ ) and a comparable decay half-life ( $3.3 \mathrm{~d}$ vs. $4.2 \mathrm{~d}$ ) to those of ${ }^{124}$ I was selected as radioisotope tracer replacing iodine-124. In order to label aMSH-PEGCy5-C ${ }^{\prime}$ dots with zirconium-89, the surface of aMSH-PEG-Cy5-C' ${ }^{\prime}$ dots were first functionalized with amine groups, using a recently developed post-PEGylation surface modification by insertion (PPSMI) approach, ${ }^{36}$ which were subsequently reacted with radiometal chelator p-SCN-Bn-deferoxamine (DFO-NCS). Sandwiching the aminefunctionalization step between PEGylation and purification steps in the $\mathrm{C}^{\prime}$ dot synthesis allowed to maintain the high-quality of the $\mathrm{C}^{\prime}$ dot synthesis. For PPSMI, aminefunctionalized silane molecules were inserted in-between the chains of the $\mathrm{C}^{\prime}$ dot PEG layer and covalently attached to the remaining silanol groups on the silica surface underneath. The resulting $\mathrm{NH}_{2}$-aMSH-PEG-Cy5-C $\mathrm{C}^{\prime}$ dots enabled further modification with amine-reactive functional ligands, e.g., DFO-NCS chelator molecules for zirconium-89. The GPC elugram of the purified $\mathrm{NH}_{2}$-aMSH-PEG-Cy5-C' ${ }^{\prime}$ dots exhibited only one peak, well fitted by a single, symmetric Gaussian distribution (Figure 2f). This indicated high purity and a narrow particle size distribution, further confirmed by TEM (inset in Figure 2f). The fit of the corresponding particle FCS correlation curve suggested an average hydrodynamic diameter of $\mathrm{NH}_{2}$-aMSH-PEG-Cy5-C $\mathrm{C}^{\prime}$ dots of $6.2 \mathrm{~nm}$ (Figure $2 \mathrm{~g}$ ). The average numbers of $\mathrm{Cy} 5$ and aMSH molecules per $\mathrm{C}^{\prime}$ dot were estimated by FCS and UV-Vis spectroscopic analysis to be 1.5 and 8.0, respectively (Figure $2 \mathrm{~h}-\mathrm{i}$ ). The $\mathrm{NH}_{2}$-aMSH-PEG-Cy5-C ${ }^{\prime}$ dots were subsequently mixed with DFO-NCS in PBS, allowing the isothiocyanate-functionalized DFO molecules to diffuse into the PEG layer of $\mathrm{NH}_{2}$-aMSH-PEG-Cy5- $\mathrm{C}^{\prime}$ dots and covalently attach to the amine groups under the PEG layer. As synthesized DFO-aMSHPEG-Cy5-C ${ }^{\prime}$ dots could then be labeled with ${ }^{89} \mathrm{Zr}$ through DFO chelation (see Experimental Section for more details). Unlike ${ }^{124} \mathrm{I}$, which is prone to dehalogenation after cellular uptake,

${ }^{89} \mathrm{Zr}$ has been reported to stably residualized within cells after its internalization, ${ }^{53}$ thereby 
enabling accurate quantitation of in vivo cellular uptake. A flow cytometry study of $\mathrm{NH}_{2}-$ aMSH-PEG-Cy5-C ${ }^{\prime}$ dots in B16F10 cells was performed to confirm that specific targeting is not substantially influenced by introducing amines to the aMSH-PEG-Cy5-C ${ }^{\prime}$ dots (as shown in Figure S6).

Prior to the PET imaging study, the biodistribution pattern of ${ }^{89} \mathrm{Zr}-\mathrm{DFO}-\mathrm{aMSH}-\mathrm{PEG}-\mathrm{Cy} 5-\mathrm{C}$ ' dots in B16F10 tumor-bearing mice was performed and compared with that of ${ }^{125} \mathrm{I}$ aMSH-PEG-Cy5-C ${ }^{\prime}$ dots in the same tumor model. As shown in Figure 6a, both radiotracers showed nearly equivalent biodistribution profiles, noting $\sim 5 \% \mathrm{ID} / \mathrm{g}$ uptake in B16F10 tumor at $24 \mathrm{~h}$ p.i., a result that likely reflects limitations in overall tumor uptake imposed by the relatively low number of MC1-R receptors expressed by this tumor as opposed to other melanoma markers (i.e., integrins). ${ }^{14,47}$ Less than $5 \% \mathrm{ID} / \mathrm{g}$ accumulation of both radio-tracers were observed in liver, spleen and kidney. Figure $6 \mathrm{~b}$ presents $\mathrm{H} \& \mathrm{E}$ staining, Cy5 fluorescence microscopy, and quantitative autoradiography of a representative ex vivo B16F10 tumor tissue specimen demonstrating excellent colocalization of optical and radiotracer signal across the specimen.

After i.v. injection of ${ }^{89} \mathrm{Zr}$-DFO-aMSH-PEG-Cy5-C' dots into B16F10 tumor-bearing mice, in vivo PET imaging (Figure 7) showed significant bladder activity $2 \mathrm{~h} \mathrm{p.i.} \mathrm{High}{ }^{89} \mathrm{Zr}$ DFO-aMSH-PEG-C ${ }^{\prime}$ dot uptake found in cardiac tissue $(10.6 \pm 1.9 \% \mathrm{ID} / \mathrm{g})$ clearly indicated significant blood pool activity. Time-activity curves, shown in Figure 8a, demonstrate the clearance of ${ }^{89} \mathrm{Zr}$-DFO-aMSH-PEG-Cy5-C' dots from the blood, with activity concentrations of $\sim 3$ and $\sim 1 \% \mathrm{ID} / \mathrm{g}$ at 24 and $72 \mathrm{~h}$ post-injection, respectively. The clearance of ${ }^{89} \mathrm{Zr}$-DFO-aMSH-PEG-Cy5-C' dots by the RES (e.g., liver) was estimated to be $5.0 \pm 0.6 \% \mathrm{ID} / \mathrm{g}$ at $2 \mathrm{~h}$ p.i., with a nearly $50 \%$ reduction (i.e., $2.3 \pm 0.2 \% \mathrm{ID} / \mathrm{g}$ ) after 3 days. Splenic uptake was found to be only half of that found in liver over the course of 3 days. Muscle uptake was low, no greater than $1 \% \mathrm{ID} / \mathrm{g}$. B16F10 tumor uptake peaked at $24 \mathrm{~h}$ postinjection at $5.5 \pm 0.9 \% \mathrm{ID} / \mathrm{g}$ (Figure $7 \mathrm{a}$ and $8 \mathrm{a}$ ), and retention of ${ }^{89} \mathrm{Zr}$-DFO-aMSH-PEG-Cy5$\mathrm{C}^{\prime}$ dots was seen over this 3-day time period (Figure 7a). As expected, mice injected with ${ }^{89} \mathrm{Zr}$-DFO-aMSH-PEG-Cy5-C ${ }^{\prime}$ dots showed minimal bone and joint uptake, indicating high in vivo radiostability (Figure 7a) (free ${ }^{89} \mathrm{Zr}^{4+}$ is an osteophilic cation, which could be readily accreted into bone mineral ${ }^{54}$ ).

To further demonstrate in vivo target specificity of ${ }^{89} \mathrm{Zr}$-DFO-aMSH-PEG-Cy5-C' dots, a blocking study was performed in a separate cohort of B16F10 tumor-bearing mice coinjected with ${ }^{89} \mathrm{Zr}$-DFO-aMSH-PEG-Cy5-C' ${ }^{\prime}$ dots and $200 \mu \mathrm{g}$ of NDP inhibitor. Results showed similar particle distributions in major organs, such as bladder, heart, liver and muscle, but with a nearly $50 \%$ reduction in tumor uptake (mean: $2-3 \% \mathrm{ID} / \mathrm{g}$ ), as shown in Figures $7 \mathrm{~b}$ and $8 \mathrm{~b}, \mathrm{c}$. For the targeted group, the highest tumor-to-blood and tumor-to-muscle ratios were estimated to be $5.8 \pm 0.7$ and $8.4 \pm 1.1$ at $72 \mathrm{~h}$ p.i., respectively, which are $\sim 2$-fold greater than the corresponding ratios obtained for the blocked group (tumor-to-blood: 3.0 0 0.6; tumor-to-muscle: $3.5 \pm 1.0$, Figures $8 \mathrm{~d}, \mathrm{e}$ ). Finally, on the basis of high tumor uptake and low RES accumulation, we observed tumor-to-liver ratios of about 2 in the targeted group (Figure 8f). These findings successfully demonstrated in vivo targeting specificity and concomitant renal clearance for ${ }^{89} \mathrm{Zr}-\mathrm{DFO}-\mathrm{aMSH}-\mathrm{PEG}-\mathrm{Cy} 5-\mathrm{C}^{\prime}$ dots in MC1-R overexpressing xenograft models. 


\section{CONCLUSION}

To address technical challenges associated with translation of potent aMSH analogs, a small library of custom-synthesized rhenium (Re)-cyclized aMSH peptide ligands were sythesized and adapted for attachment to ultrasmall fluorescent silica nanoparticles. The resulting MC1-R particle probes were shown to have subnanomolar receptor affinity and rapid receptor-mediated internalization in melanoma cells. In vivo, radiolabeled aMSH-PEGCy5- $\mathrm{C}^{\prime}$ dots displayed favorable pharmacokinetics and dosimetry, highlighted by their accumulation and retention in MC1-R-expressing tumors, efficient renal excretion, and low RES uptake. Ex vivo, tumor penetration and distribution of aMSH-PEG-Cy5-C ${ }^{\prime}$ dots were visualized by $\mathrm{Cy} 5$ fluorescence imaging and autoradiography. Site-specific uptake in B16F10 xenografts was assessed by blocking with excess NDP peptide using PET imaging and ${ }^{89} \mathrm{Zr}$ - and ${ }^{124} \mathrm{I}$-labeled aMSH-PEG-Cy5-C' dots. These results highlight the promising clinical potential of aMSH-PEG-Cy5-C' dots as dual-modality particle probes for the image-guided surgical treatment and targeted radiotherapy of melanoma by labeling aMSHPEG-Cy5-C ${ }^{\prime}$ dots with alpha and beta emitting radionuclides.

\section{MATERIALS AND METHODS}

\section{General Synthesis procedures of Peptides}

Three MC1-R targeting ReCCMSH peptide analogs (Figure S1) were synthesized using standard fluorenylmethyloxycarbonyl (Fmoc) solid-phase peptide chemistry on a multiple peptide synthesizer (Model 396, AAPPTEC or Tetras, Advanced ChemTech). ${ }^{37} 4-$ Methyltrityl (Mmt) protection groups were selectively removed from the cysteines involved in peptide cyclization by treatment with $1 \%$ trifluoroacetic acid (TFA) and 5\% triisopropylsilane (TIS) in dichloromethane (DCM). Rhenium (Re) cyclization was achieved directly on solid phase by overnight reaction with 4 molar excess of $\mathrm{ReOCl}_{3}\left(\mathrm{PPh}_{2}\right)_{3}$ in Nmethyl-2-pyrrolidone (NMP). Cleavage and sidechain deprotection were achieved by treating the peptidyl-resin with $85 \%$ TFA and non-reducing scavengers (phenol/water/TIS, $5 \%$ each). The peptides were purified and analyzed on a Beckman Coulter High Performance Liquid Chromatography (HPLC) system coupled with an LCQ FLEET Ion Trap Mass spectrometer (MS; Thermo Fisher Scientific). The final products were recovered by lyophilization and stored at $-20^{\circ} \mathrm{C}$.

\section{Synthesis of aMSH peptide I}

Peptide I ( $\mathrm{NH}_{2}$ - $\mathrm{Re}$ [Cyc-Cys-Glu-His-dPhe-Arg-Trp-Cys]-Arg-Pro-Val-NH 2 (Figure S1a) was synthesized, as described above. The purified peptide was subjected to HPLC MS analysis; calculated and experimental mass-to-charge (m/z) ratios were 1632 and 1633 , respectively. Peptide I was modified at both termini, first with $\mathrm{N}$-succinimidyl Sacetylthiolacetate (SATA) reagent, to convert one amine to a sulfhydryl; this was followed by a second amine modification to introduce a N-succinimidyl-3-(4-hydroxyphenyl) propionate (SHPP; Bolton-Hunter reagent) group for radioiodination. Peptide I was reacted with SATA reagent at a 9:1 SATA-to-peptide molar ratio in phosphate buffered saline (PBS) $\mathrm{pH} 7.4$ at $25^{\circ} \mathrm{C}$ for $30 \mathrm{~min}$. The reaction was quenched by the addition of $1 \mathrm{mM}$ ethanolamine and purified over a size exclusion column. The acetylthioacetate-modified 
peptide I was reacted with SHPP at a SHPP-to-peptide ratio of 10:1 in $200 \mathrm{mM}$ borate buffer (pH 9.0) on ice for $3 \mathrm{~h}$. Modified peptides were purified from unreacted SHPP by size exclusion chromatography. The sulfhydryl acetyl protection group was removed with $0.5 \mathrm{M}$ hydroxylamine and $25 \mathrm{mM}$ EDTA in PBS (pH 7.4) for $2 \mathrm{~h}$ at $25^{\circ} \mathrm{C}$, and purified on a G25 size exclusion column to yield the final HPP-Peptide I-thiolacetate.

\section{Synthesis of aMSH peptide II}

Peptide II ( $\mathrm{NH}_{2}$-Ser-Tyr-Ser-Re[Cys-Cys-Glu-His-dPhe-Arg-Trp-Cys]-Arg-Pro-Val- $\mathrm{NH}_{2}$ (Figure S1b) was synthesized using standard solid-phase Fmoc peptide synthesis, and modified with SATA reagent for the introduction of a terminal sulfhydryl as described above. The purified peptide was subjected to HPLC MS analysis; m/z, calculated 1972, experimental 1972 .

\section{Synthesis of aMSH peptide III}

Peptide III (Ac-Cys-(Ahx) 2 $_{2}$-dLys-Re[Cys-Cys-Glu-His-dPhe-Arg-Trp-Cys]-Arg-Pro-Val$\mathrm{NH}_{2}$ (aMSH, Figure S1c) was synthesized with a $\mathrm{N}$-acetylated-cysteine, a double aminohexanoic acid $\left(\mathrm{Ahx}_{2}\right)$ aliphatic linker and a dLys using standard solid-phase Fmoc peptide chemistry, as described above. The purified peptide was subjected to HPLC MS analysis; m/z, calculated 2131, experimental 2131.

\section{Synthesis of scrambled (Scr) MSH peptide}

The Scr-MSH peptide control, Ac-Cys-(Ahx) $)_{2}$-D-Lys-Re[Cys-Cys-Trp-Arg-His-dPhe-GluCys]-Arg-Pro-Val- $\mathrm{NH}_{2}$ was synthesized using standard solid phase peptide synthesis chemistry. It contained the same $\mathrm{N}$-terminal Ac-Cys, $(\mathrm{Ahx})_{2}$ linker and D-Lys as aMSH peptide III. Only the Re-cyclized MC1-R binding sequence was scrambled. The purified peptide was subjected to HPLC MS analysis; m/z, calculated 2131, experimental 2131.

\section{Synthesis of aMSH-PEG-Cy5-C' dots and $\mathrm{NH}_{2}-\mathrm{aMSH}-\mathrm{PEG}-\mathrm{Cy} 5-\mathrm{C}^{\prime}$ dots}

The aMSH-PEG-Cy5-C' dots were synthesized based on a previously published protocol. ${ }^{32}$ More specifically, NHS ester/maleimido heterobifunctional polyethylene glycol (PEG) with molar mass around 860 (12 ethylene glycol units) was first conjugated with (3aminopropyl)triethoxysilane at room temperature under nitrogen through amine-NHS ester reaction, forming mal-PEG-silane. aMSH was then added into the mixture at room temperature under nitrogen to further conjugate mal-PEG-silane with aMSH through thiolene reaction, forming aMSH-PEG-silane. At the same time, Cy5-silane conjugate was prepared by conjugating maleimido functionalized Cy5 dyes with (3mercaptopropyl)trimethoxysilane at room temperature under nitrogen. In the next step, tetramethyl orthosilicate (TMOS) and Cy5-silane were added to an aqueous solution with ammonium hydroxide adjusting the $\mathrm{pH}$ around 8.5 at room temperature under vigorous stirring. One day later, aMSH-PEG-silane and monofunctional PEG-silane with molar mass around 500 (6 to 9 ethylene glycol units) were added into the reaction at room temperature under vigorous stirring. The reaction mixture was further left at room temperature under vigorous stirring overnight, followed by an overnight high temperature treatment at $80{ }^{\circ} \mathrm{C}$ without stirring. After cooling the reaction to room temperature, the resulting aMSH-PEG- 
Cy5-C ${ }^{\prime}$ dots were dialyzed in DI water, purified using GPC, filtered by sterile syringe filters and finally stored at $4{ }^{\circ} \mathrm{C}$. The full name of the aMSH-PEG-Cy5-C' dots using a comprehensive nomenclature introduced in a recent publication to differentiate between different $\mathrm{C}^{\prime}$ dot chemistries is CD(Cy5)-PEG12-mal-thiol-aMSH_PEG6. ${ }^{36}$

The synthesis of $\mathrm{NH}_{2}$-aMSH-PEG-Cy5- $\mathrm{C}^{\prime}$ dots followed a similar protocol as described above, in which a PPSMI approach was employed to functionalize aMSH-PEG-Cy5-C' dots with amine groups. ${ }^{36}$ More specifically, after the reaction temperature of the postPEGylation heat treatment was reduced from $80{ }^{\circ} \mathrm{C}$ to room temperature, (3aminopropyl)trimethoxysilane was added at room temperature under vigorous stirring. The reaction solution was left at room temperature under vigorous stirring overnight to insert the small amine-silane molecules in-between the PEG layer of aMSH-PEG-Cy5-C ${ }^{\prime}$ dots and to covalently attach them to the silanol groups of the underlying silica particle surface. The resulting $\mathrm{NH}_{2}$-aMSH-PEG-Cy5-C $\mathrm{C}^{\prime}$ dots were dialyzed in DI water, purified using GPC, filtered by sterile syringe filters and finally stored at $4{ }^{\circ} \mathrm{C}$. The full name of the $\mathrm{NH}_{2}$ - $\mathrm{aMSH}$ PEG-Cy5- $\mathrm{C}^{\prime}$ dots using the comprehensive nomenclature described above is $\mathrm{CD}(\mathrm{Cy} 5)$ PEG12-mal-thiol-aMSH_PEG6_NH $2 .{ }^{36}$

GPC purification and characterization of the particles was conducted using a biologic LP system (Bio-Rad) equipped with a $275 \mathrm{~nm}$ UV detector and a chromatography column packed with Superdex 200 resin from GE healthcare. TEM images were taken on an FEI T12 Spirit TEM operated at an acceleration voltage of $120 \mathrm{kV}$. UV-Vis absorbance spectra were measured on a Varian Cary 5000 spectrophotometer. FCS measurements were conducted using a home-built FCS setup with a $633 \mathrm{~nm}$ solid state laser as the excitation source.

\section{Cells and cell culture}

Human melanoma M21 was obtained from D.A. Cheresh (University of California San Diego, San Diego, California, USA). Cells were maintained in RPMI 1640 media/10\% FBS, and $2 \mathrm{mM}$ l-glutamine, penicillin, and streptomycin (Core Media Preparation Facility, Memorial Sloan Kettering Cancer Center). B16F10 cells were obtained from American Type Culture Collection (ATCC). Cells were maintained in RPMI 1640 supplemented with 10\% fetal bovine serum (FBS), 2 mM L-glutamine and Penicillin Streptomycin.

\section{In vitro MC1-R-overexpressing cell binding studies}

To assay particle binding in MC1-R-overexpressing cell lines, 24-well plates were coated with $10 \mu \mathrm{g} / \mathrm{ml}$ collagen type I (BD Biosciences) in PBS, incubated at $37^{\circ} \mathrm{C}$ for 30 minutes, and washed once with PBS. M21 and B16F10 cells $\left(3.0 \times 10^{5}-4.0 \times 10^{5}\right.$ cells/well) were grown to confluency. Differential binding of aMSH-PEG-Cy5-C' dots to M21 or B16F10 cells was evaluated over a range of incubation times ( $0-5$ hours) and particle concentrations (10-300 nM). After incubation, cells were washed with RPMI 1640 media/0.5\% BSA, detached using $0.25 \%$ trypsin/EDTA, pelleted in a microcentrifuge tube (5 minutes at $153 \mathrm{~g}$, $25^{\circ} \mathrm{C}$ ), re-suspended in BD FACSFlow solution (BD Biosciences), and analyzed by flow cytometry in the Cy-5 channel to determine the percentage of particle-bound probe (FACSCalibur, Becton Dickinson, Mountain View, CA). Blocking studies were performed 
after incubation of aMSH-PEG-Cy5-C' dots with M21 cells (or B16F10) in the presence of Anti-MC1 Receptor antibody (EPR6530, ab125031, http://www.abcam.com/mc1-receptorantibody-epr6530-ab125031.html), and analyzed by flow cytometry. Binding studies were also conducted in B16F10 cells using a particle control (i.e., Scr-MSH-PEG-Cy5-C' dots, $25 \mathrm{nM})$.

\section{Cellular internalization and microscopic imaging}

M21 melanoma cells expressing LAMP1-GFP were generated by retroviral transduction of a pRetro-Lamp1-GFP construct and selection in puromycin $(2 \mu \mathrm{g} / \mathrm{ml})$. Cells were then cultured overnight on glass-bottom 35mm dishes (MatTek, Ashland, MA), incubated with $1.5 \mu \mathrm{M}$ aMSH-PEG-C ${ }^{\prime}$ dots for 6 hours, and imaged by widefield microscopy, using a Nikon Ti-E inverted microscope and a CoolSnap HQ2 CCD camera (Photometrics, Tucson, AZ). Images were processed with Elements software (Nikon, Melville, NY) and ImageJ software.

\section{Cell Viability and Proliferation}

Viability and proliferation of M21 and B16F10 cells were monitored under control conditions $(10 \% \mathrm{FBS})$ or after exposure to particles at $37^{\circ} \mathrm{C}$. Cells were split $\left(4-5 \times 10^{4}\right.$ cells/ well) in a 24-well plate coated with collagen, as described above. Media was changed to RPMI, supplemented with $10 \%$ FBS, $24 \mathrm{~h}$ later, and different concentrations of aMSHPEG-C ${ }^{\prime}$ dots were added $(25-250 \mathrm{nM})$ for $24-48$ hours at $37^{\circ} \mathrm{C}$. The percent (\%) cell viability was determined 24 or $48 \mathrm{~h}$ post-incubation after trypsinization using a Vi-Cell series viability analyzer (Beckman Coulter, Inc, Fullerton, CA). To assess proliferative activity, particle-exposed cells were counted every $24 \mathrm{~h}$ over a 96-h time interval.

\section{Competitive binding assay $\left(\mathrm{IC}_{50}\right)$}

The $\mathrm{IC}_{50}$ (concentration of peptide required to inhibit $50 \%$ of radioligand binding) was determined for aMSH-PEG-Cy5-C' dots and Scr-MSH-PEG-Cy5-C ${ }^{\prime}$ dots in competitive binding assays with ${ }^{125} \mathrm{I}-\left(\mathrm{Tyr}^{2}\right)$-NDP, a radioiodinated aMSH analog with picomolar affinity for the MC1-R receptor. ${ }^{55}$ Murine melanoma B16F10 cells were plated at a density of 0.2 million/well in 24-well tissue culture plates and incubated overnight. Individual wells were incubated at $25^{\circ} \mathrm{C}$ for $3 \mathrm{~h}$ with approximately $50,000 \mathrm{cpm}(62,480 \mathrm{dpm}){ }^{125} \mathrm{I}_{-}\left(\mathrm{Tyr}^{2}\right)-\mathrm{NDP}$ in $0.3 \mathrm{~mL}$ binding medium (DMEM with $25 \mathrm{mM} \mathrm{N}$-(2-hydroxyethyl)-piperazine-N'-(2ethanesulfonic acid) (HEPES), pH 7.4, 0.02\% BSA, and $0.3 \mathrm{mM}$ 1,10-phenanthroline) with concentrations of the aMSH-PEG-Cy5-C' ${ }^{\prime}$ dots and Scr-MSH-PEG-Cy5- $\mathrm{C}^{\prime}$ dots ranging from $10^{-13}$ to $10^{-5} \mathrm{~mol} / \mathrm{L}$. The binding media was aspirated after incubation. Cells were rinsed with $0.5 \mathrm{~mL}$ of ice-cold $\mathrm{pH} 7.1,0.02 \% \mathrm{BSA} / 0.01 \mathrm{M}$ PBS twice and lysed with 0.5 $\mathrm{mL}$ of $1 \mathrm{M} \mathrm{NaOH}$ for $10 \mathrm{~min}$. The radioactivity in the cells was collected and measured in a $\gamma$-counter. The $\mathrm{IC}_{50}$ values of aMSH-PEG-Cy5-C' dots and Scr-MSH-PEG-Cy5-C' dots were calculated with GraphPad Prism version 4.0.

\section{Animal models and tumor inoculation}

All animal experiments were done in accordance with protocols approved by the Institutional Animal Care and Use Committee of Memorial Sloan-Kettering Cancer Center and followed NIH guidelines for animal welfare. M21 and B16F10 xenografts were 
generated by co-injecting equal volumes of cells $\left(\sim 5 \times 10^{6}\right.$ cells $\left./ 100 \mu \mathrm{L}\right)$ and Matrigel subcutaneously into the hind legs of female athymic nu/nu mice (6-8 weeks old, Taconic Farms Inc.). Average tumor volumes of $200 \mathrm{~mm}^{3}$ were used for all studies.

\section{I radiolabeling of aMSH-PEG- and Scr-MSH-PEG-Cy5-C' dots for cell binding and biodistribution studies}

$1.8 \mathrm{mCi}$ of ${ }^{125}$ I Bolton Hunter reagent (PerkinElmer) was evaporated to dryness in a glass vial with $\mathrm{N}_{2}$ gas, followed by the addition of $100 \mu \mathrm{L}$ of DMSO, $15 \mu \mathrm{L}$ of DIEA and an equal molar concentration of aMSH-PEG-Cy5- $\mathrm{C}^{\prime}$ dots or Scr-MSH-PEG-Cy5-C $\mathrm{C}^{\prime}$ dots $(15 \mu \mathrm{M}$, $<10 \mathrm{MSH})$. The mixture was kept at room temperature for 30 minutes before it was loaded into a pre-conditioned PD10 column and eluted with $0.5 \mathrm{~mL}$ of saline. The eluent was collected for biodistribution studies in B16/F10 and M21 melanoma-bearing mice. The yield was approximately $7 \%$, and product specific activity was $3100 \mathrm{Ci} / \mathrm{mol} .{ }^{125} \mathrm{I}$-aMSH-PEG-C ${ }^{\prime}$ dots $(\sim 50 \mu \mathrm{Ci}$, or $\sim 1.85 \mathrm{MBq})$ were i.v.-injected in both tumor models.

\section{${ }^{124}$ I radiolabeling of aMSH-PEG-C'dots for a pilot PET/CT imaging}

Ten times molar excess of $\mathrm{N}$-succinimidyl-3-(4-hydroxyphenyl) propionate (Bolton-Hunter Reagent; SHPP) was added to aMSH-PEG-Cy5-C ${ }^{\prime}$ dots $(15 \mu \mathrm{M})$ in $0.2 \mathrm{M}$ Borax (pH 8.7), vortexed, and allowed to proceed at room temperature (RT) shielded from light for 3 hours. The mixture was dialyzed in triplicate using a 10,000 molecular weight cut-off membrane (Thermo Scientific) against phosphate-buffered saline (PBS, 0.1 M sodium phosphate, $150 \mathrm{mM}$ sodium chloride, $\mathrm{pH}$ 7.2). Equal molar amounts of 3-(4-hydroxyphenyl) propionate (HPP)-aMSH-PEG-C ${ }^{\prime}$ dots and ${ }^{124}$ I were then added into iodogen-coated $(20 \mu \mathrm{g})$ vials containing PBS; the total volume was $100 \mu \mathrm{L}$. After vortexing, the reaction was allowed to proceed at RT for 20 minutes. The radioiodinated product was purified four times with a Zeba spin column $(7 \mathrm{~K})$. The yield was $25 \%$, and product specific activity was $5.5 \times 10^{6} \mathrm{Ci} /$ mol. The radiochemical purity was greater than $92 \%$ using ITLC. For the mobile phase, $\mathrm{MeOH}$ : Ethyl Acetate (1:1) was used; Rf values for the particle tracer ( ${ }^{124} \mathrm{I}-\mathrm{aMSH}-\mathrm{PEG}-\mathrm{C}^{\prime}$ dots) and free radioiodine $\left({ }^{124} \mathrm{I}^{-}\right)$were 0 and $0.7-0.8$, respectively.

\section{${ }^{89} \mathrm{Zr}$-oxalate production}

${ }^{89} \mathrm{Zr}$ was produced at Memorial Sloan Kettering Cancer Center on a TR19/9 cyclotron (Ebco Industries Inc.) via the ${ }^{89} \mathrm{Y}(\mathrm{p}, \mathrm{n}){ }^{89} \mathrm{Zr}$ reaction, and purified to yield ${ }^{89} \mathrm{Zr}$ with a specific activity of $5.28-13.43 \mathrm{mCi} / \mu \mathrm{g}(470-1195 \mathrm{Ci} / \mathrm{mmol})$ of zirconium. ${ }^{56}$ Activity measurements were performed using a CRC-15R Dose Calibrator (Capintec). For the quantification of activities, experimental samples were counted on an Automatic Wizard ${ }^{2} \gamma$-Counter (PerkinElmer). All in vivo experiments were performed according to protocols approved by the Memorial Sloan Kettering Institutional Animal Care and Use Committee. A purity of greater than $95 \%$ was confirmed using radio-TLC for all of the ${ }^{89} \mathrm{Zr}$-labeled DFO-aMSHPEG-Cy5-C' dots.

\section{Synthesis of DFO-aMSH-PEG-Cy5-C' dots and ${ }^{89} \mathrm{Zr}$ radiolabeling}

The conjugation of DFO to $\mathrm{NH}_{2}$-aMSH-PEG-Cy5-C' dots was achieved by following a previously reported protocol with slight modifications. ${ }^{57} \mathrm{NH}_{2}$-aMSH-PEG-Cy5-C' dots 
( $200 \mu \mathrm{L}, 15 \mu \mathrm{M}$ ) in DI water were first added to $400 \mu \mathrm{L}$ PBS (pH 7.4), followed by adding DFO-NCS (30 nmol in DMSO). The final $\mathrm{pH}$ was adjusted to 8-9. The reaction was kept at RT with agitation $\left(640 \mathrm{rpm}\right.$ ) for $2 \mathrm{~h}$. About 2-3 DFO per aMSH-PEG-C' ${ }^{\prime}$ dot was estimated. To remove the un-reacted DFO-NCS, as-synthesized DFO-aMSH-PEG-Cy5-C' dots were then purified by passing through a PD-10 column using PBS as the mobile phase.

To label DFO-aMSH-PEG-Cy5-C ${ }^{\prime}$ dots with ${ }^{89} \mathrm{Zr}, 0.75$ nmol of DFO-aMSH-PEG-Cy5-C' dots were mixed with $1 \mathrm{mCi}$ of ${ }^{89} \mathrm{Zr}$-oxalate in HEPES buffer (pH 8) at $37^{\circ} \mathrm{C}$ for $60 \mathrm{~min}$; final labeling $\mathrm{pH}$ was kept as 7-7.5. An EDTA challenge process was introduced to remove any unconjugated ${ }^{89} \mathrm{Zr}$. The final ${ }^{89} \mathrm{Zr}$ labeling yield was on the order of 70 to $80 \%(\mathrm{n}>5)$. As-synthesized ${ }^{89} \mathrm{Zr}$-DFO-aMSH-PEG-Cy5-C' dots were then purified using a PD-10 column. The final radiochemical purity was estimated to be greater than $99 \%$ by ITLC.

\section{Ex vivo biodistribution and in vivo tumor-targeting PET imaging studies}

For biodistribution studies, B16F10 tumor-bearing mice $(\mathrm{n}=5)$ were i.v.-injected with 100$150 \mu \mathrm{Ci}(3.7-5.55 \mathrm{MBq}){ }^{89} \mathrm{Zr}-\mathrm{DFO}-\mathrm{aMSH}-\mathrm{PEG}-\mathrm{Cy} 5-\mathrm{C}^{\prime}$ dots, and major organs/tissues harvested and weighed $24 \mathrm{~h}$ post-injection. Accumulated activity was counted using an Automatic Wizard ${ }^{2} \gamma$-Counter (PerkinElmer), and presented as \%ID/g (mean \pm s.d.).

For PET imaging studies, B16F10 tumor-bearing mice $(\mathrm{n}=3)$ were $i . v$. injected with 200-300 $\mu \mathrm{Ci}(7.4-11.1 \mathrm{MBq}){ }^{89} \mathrm{Zr}$-DFO-aMSH-PEG-Cy5-C' dots and serially imaged in a smallanimal PET scanner (Focus 120 microPET; Concorde Microsystems) at 2, 24, 48, and $72 \mathrm{~h}$ post-injection. An energy window of 350-700 keV and a coincidence timing window of $6 \mathrm{~ns}$ were used. Data were sorted into 2D histograms by Fourier rebinning, and transverse images were reconstructed by filtered back-projection into a $128 \times 128 \times 63(0.72 \times 0.72 \times 1.3$ $\mathrm{mm}^{3}$ ) matrix. The PET imaging data was normalized to correct for non-uniformity of response, dead-time count losses, positron branching ratio, and physical decay to the time of injection; no attenuation, scatter, or partial-volume averaging corrections were applied. The counting rates in the reconstructed images were converted to activity concentrations (percentage injected dose per gram of tissue, $\% \mathrm{ID} / \mathrm{g}$ ) by use of a system calibration factor derived from the imaging of a mouse-sized water-equivalent phantom containing ${ }^{89} \mathrm{Zr}$. Region-of-interest (ROI) analyses of the PET data were performed using IRW software. Blocking studies were performed by co-injecting mice $(n=3)$ with similar amounts of ${ }^{89} \mathrm{Zr}$ DFO-aMSH-PEG-Cy5-C' dots and $200 \mathrm{mg}$ NDP.

\section{Digital Autoradiography}

$10 \mu \mathrm{m}$ B16F10 tumor tissue sections were exposed to phosphor-imaging plates (Fujifilm BAS-MS2325, Fuji Photo Film, Japan) at $-20^{\circ} \mathrm{C}$. Following exposure, the phosphor-imaging plate was read at a resolution of $25 \mu \mathrm{m}$ using a Typhoon $7000 \mathrm{IP}$ (GE Life Sciences) plate reader to produce digital autoradiograms. Images were saved as both TIFF and GEL formats for future analysis and processing with ImageJ software (https://imagej.nih.gov/ij/).

\section{Statistics}

All comparisons were based on statistical significance from pairwise t-tests from an analysis of variance model based on three replicates. 


\section{Supplementary Material}

Refer to Web version on PubMed Central for supplementary material.

\section{Acknowledgments}

\section{Funding Sources}

This study was funded by grants from the National Institutes of Health (1R01CA161280-01A1 to M.B. and U.W.; 1U54 CA199081-01 to M.B. and U.W.); and Sloan Kettering Institute (Core Grant P30 CA008748CCSG). University of Missouri Peptide Synthesis Core and Harry S Truman VA Hospital Biomolecular Imaging Center and Research Animal Core.

\section{References}

1. Society, A. C. Cancer Facts \& Figures 2016. American Cancer Society; 2017.

2. Chen KG, Valencia JC, Gillet JP, Hearing VJ, Gottesman MM. Involvement of Abc Transporters in Melanogenesis and the Development of Multidrug Resistance of Melanoma. Pigment Cell Melanoma Res. 2009; 22:740-749. [PubMed: 19725928]

3. Roesch A, Vultur A, Bogeski I, Wang H, Zimmermann KM, Speicher D, Korbel C, Laschke MW, Gimotty PA, Philipp SE, Krause E, Patzold S, Villanueva J, Krepler C, Fukunaga-Kalabis M, Hoth M, Bastian BC, Vogt T, Herlyn M. Overcoming Intrinsic Multidrug Resistance in Melanoma by Blocking the Mitochondrial Respiratory Chain of Slow-Cycling Jarid1b(High) Cells. Cancer Cell. 2013; 23:811-825. [PubMed: 23764003]

4. Sabatino M, Stroncek DF, Klein H, Marincola FM, Wang E. Stem Cells in Melanoma Development. Cancer Lett. 2009; 279:119-125. [PubMed: 19095348]

5. Salama AK. Evolving Pharmacotherapies for the Treatment of Metastatic Melanoma. Clin Med Insights Oncol. 2013; 7:137-149. [PubMed: 23843723]

6. Robert C, Thomas L, Bondarenko I, O'Day S, Weber J, Garbe C, Lebbe C, Baurain JF, Testori A, Grob JJ, Davidson N, Richards J, Maio M, Hauschild A, Miller WH Jr, Gascon P, Lotem M, Harmankaya K, Ibrahim R, Francis S, Chen TT, Humphrey R, Hoos A, Wolchok JD. Ipilimumab Plus Dacarbazine for Previously Untreated Metastatic Melanoma. N Engl J Med. 2011; 364:25172526. [PubMed: 21639810]

7. Hodi FS, O'Day SJ, McDermott DF, Weber RW, Sosman JA, Haanen JB, Gonzalez R, Robert C, Schadendorf D, Hassel JC, Akerley W, van den Eertwegh AJ, Lutzky J, Lorigan P, Vaubel JM, Linette GP, Hogg D, Ottensmeier CH, Lebbe C, Peschel C, Quirt I, Clark JI, Wolchok JD, Weber JS, Tian J, Yellin MJ, Nichol GM, Hoos A, Urba WJ. Improved Survival with Ipilimumab in Patients with Metastatic Melanoma. N Engl J Med. 2010; 363:711-723. [PubMed: 20525992]

8. Topalian SL, Hodi FS, Brahmer JR, Gettinger SN, Smith DC, McDermott DF, Powderly JD, Carvajal RD, Sosman JA, Atkins MB, Leming PD, Spigel DR, Antonia SJ, Horn L, Drake CG, Pardoll DM, Chen L, Sharfman WH, Anders RA, Taube JM, McMiller TL, Xu H, Korman AJ, JureKunkel M, Agrawal S, McDonald D, Kollia GD, Gupta A, Wigginton JM, Sznol M. Safety, Activity, and Immune Correlates of Anti-Pd-1 Antibody in Cancer. N Engl J Med. 2012; 366:2443-2454. [PubMed: 22658127]

9. Robert C, Ribas A, Wolchok JD, Hodi FS, Hamid O, Kefford R, Weber JS, Joshua AM, Hwu WJ, Gangadhar TC, Patnaik A, Dronca R, Zarour H, Joseph RW, Boasberg P, Chmielowski B, Mateus C, Postow MA, Gergich K, Elassaiss-Schaap J, Li XN, Iannone R, Ebbinghaus SW, Kang SP, Daud A. Anti-Programmed-Death-Receptor-1 Treatment with Pembrolizumab in Ipilimumab-Refractory Advanced Melanoma: A Randomised Dose-Comparison Cohort of a Phase 1 Trial. Lancet. 2014; 384:1109-1117. [PubMed: 25034862]

10. Wolchok JD, Kluger H, Callahan MK, Postow MA, Rizvi NA, Lesokhin AM, Segal NH, Ariyan CE, Gordon RA, Reed K, Burke MM, Caldwell A, Kronenberg SA, Agunwamba BU, Zhang X, Lowy I, Inzunza HD, Feely W, Horak CE, Hong Q, Korman AJ, Wigginton JM, Gupta A, Sznol M. Nivolumab Plus Ipilimumab in Advanced Melanoma. N Engl J Med. 2013; 369:122-133. [PubMed: 23724867] 
11. Sullivan RJ, Flaherty KT. Resistance to Braf-Targeted Therapy in Melanoma. Eur J Cancer. 2013; 49:1297-1304. [PubMed: 23290787]

12. Tatro JB, Reichlin S. Specific Receptors for Alpha-Melanocyte-Stimulating Hormone Are Widely Distributed in Tissues of Rodents. Endocrinology. 1987; 121:1900-1907. [PubMed: 2822378]

13. Siegrist W, Solca F, Stutz S, Giuffre L, Carrel S, Girard J, Eberle AN. Characterization of Receptors for Alpha-Melanocyte-Stimulating Hormone on Human Melanoma Cells. Cancer Res. 1989; 49:6352-6358. [PubMed: 2804981]

14. Miao Y, Whitener D, Feng W, Owen NK, Chen J, Quinn TP. Evaluation of the Human Melanoma Targeting Properties of Radiolabeled Alpha-Melanocyte Stimulating Hormone Peptide Analogues. Bioconjug Chem. 2003; 14:1177-1184. [PubMed: 14624632]

15. Miao Y, Quinn TP. Alpha-Melanocyte Stimulating Hormone Peptide-Targeted Melanoma Imaging. Front Biosci. 2007; 12:4514-4524. [PubMed: 17485393]

16. Raposinho PD, Correia JD, Oliveira MC, Santos I. Melanocortin-1 Receptor-Targeting with Radiolabeled Cyclic Alpha-Melanocyte-Stimulating Hormone Analogs for Melanoma Imaging. Biopolymers. 2010; 94:820-829. [PubMed: 20564045]

17. Quinn T, Zhang X, Miao Y. Targeted Melanoma Imaging and Therapy with Radiolabeled AlphaMelanocyte Stimulating Hormone Peptide Analogues. G Ital Dermatol Venereol. 2010; 145:245258. [PubMed: 20467398]

18. Eberle AN, Rout B, Qi MB, Bigliardi PL. Synthetic Peptide Drugs for Targeting Skin Cancer: Malignant Melanoma and Melanotic Lesions. Curr Med Chem. 2017; 24:1797-1826. [PubMed: 28578648]

19. Nasti TH, Timares L. Mc1r, Eumelanin and Pheomelanin: Their Role in Determining the Susceptibility to Skin Cancer. Photochem Photobiol. 2015; 91:188-200. [PubMed: 25155575]

20. Hruby VJ. Designing Peptide Receptor Agonists and Antagonists. Nat Rev Drug Discov. 2002; 1:847-858. [PubMed: 12415245]

21. Sawyer TK, Sanfilippo PJ, Hruby VJ, Engel MH, Heward CB, Burnett JB, Hadley ME. 4Norleucine, 7-D-Phenylalanine-Alpha-Melanocyte-Stimulating Hormone: A Highly Potent AlphaMelanotropin with Ultralong Biological Activity. Proc Natl Acad Sci U S A. 1980; 77:5754-5758. [PubMed: 6777774]

22. Garg PK, Alston KL, Welsh PC, Zalutsky MR. Enhanced Binding and Inertness to Dehalogenation of Alpha-Melanotropic Peptides Labeled Using N-Succinimidyl 3-Iodobenzoate. Bioconjug Chem. 1996; 7:233-239. [PubMed: 8983345]

23. Giblin MF, Jurisson SS, Quinn TP. Synthesis and Characterization of Rhenium-Complexed AlphaMelanotropin Analogs. Bioconjug Chem. 1997; 8:347-353. [PubMed: 9177840]

24. Giblin MF, Wang N, Hoffman TJ, Jurisson SS, Quinn TP. Design and Characterization of AlphaMelanotropin Peptide Analogs Cyclized through Rhenium and Technetium Metal Coordination. Proc Natl Acad Sci U S A. 1998; 95:12814-12818. [PubMed: 9788997]

25. Guo H, Gallazzi F, Miao Y. Gallium-67-Labeled Lactam Bridge-Cyclized Alpha-Msh Peptides with Enhanced Melanoma Uptake and Reduced Renal Uptake. Bioconjug Chem. 2012; 23:13411348. [PubMed: 22621181]

26. Miao Y, Fisher DR, Quinn TP. Reducing Renal Uptake of 90y- and 177lu-Labeled AlphaMelanocyte Stimulating Hormone Peptide Analogues. Nucl Med Biol. 2006; 33:723-733. [PubMed: 16934691]

27. Bapst JP, Froidevaux S, Calame M, Tanner H, Eberle AN. Dimeric Dota-Alpha-MelanocyteStimulating Hormone Analogs: Synthesis and in Vivo Characteristics of Radiopeptides with High in Vitro Activity. J Recept Signal Transduct Res. 2007; 27:383-409. [PubMed: 18097939]

28. Lin CH, Al-Suwayeh SA, Hung CF, Chen CC, Fang JY. Camptothecin-Loaded Liposomes with Alpha-Melanocyte-Stimulating Hormone Enhance Cytotoxicity toward and Cellular Uptake by Melanomas: An Application of Nanomedicine on Natural Product. J Tradit Complement Med. 2013; 3:102-109. [PubMed: 24716164]

29. Barkey NM, Tafreshi NK, Josan JS, De Silva CR, Sill KN, Hruby VJ, Gillies RJ, Morse DL, Vagner J. Development of Melanoma-Targeted Polymer Micelles by Conjugation of a Melanocortin 1 Receptor (Mc1r) Specific Ligand. J Med Chem. 2011; 54:8078-8084. [PubMed: 22011200] 
30. Lu W, Xiong C, Zhang R, Shi L, Huang M, Zhang G, Song S, Huang Q, Liu G, Li C. ReceptorMediated Transcytosis: A Mechanism for Active Extravascular Transport of Nanoparticles in Solid Tumors. J Control Release. 2012; 161:959-966. [PubMed: 22617522]

31. Lu W, Xiong C, Zhang G, Huang Q, Zhang R, Zhang JZ, Li C. Targeted Photothermal Ablation of Murine Melanomas with Melanocyte-Stimulating Hormone Analog-Conjugated Hollow Gold Nanospheres. Clin Cancer Res. 2009; 15:876-886. [PubMed: 19188158]

32. Ma K, Mendoza C, Hanson M, Werner-Zwanziger U, Zwanziger J, Wiesner U. Control of Ultrasmall Sub-10 Nm Ligand-Functionalized Fluorescent Core-Shell Silica Nanoparticle Growth in Water. Chem Mater. 2015; 27:4119-4133.

33. Ma K, Zhang DH, Cong Y, Wiesner U. Elucidating the Mechanism of Silica Nanoparticle Pegylation Processes Using Fluorescence Correlation Spectroscopies. Chem Mater. 2016; 28:1537-1545.

34. Chen F, Ma K, Benezra M, Zhang L, Cheal SM, Phillips E, Yoo B, Pauliah M, Overholtzer M, Zanzonico P, Sequeira S, Gonen M, Quinn T, Wiesner U, Bradbury MS. Cancer-Targeting Ultrasmall Silica Nanoparticles for Clinical Translation: Physicochemical Structure and Biological Property Correlations. Chem Mater. 2017; doi: 10.1021/acs.chemmater.1027b03033

35. Piao Y, Burns A, Kim J, Wiesner U, Hyeon T. Designed Fabrication of Silica-Based Nanostructured Particle Systems for Nanomedicine Applications. Adv Funct Mater. 2008; 18:3745-3758.

36. Ma K, Wiesner U. Modular and Orthogonal Post-Pegylation Surface Modifications by Insertion Enabling Penta-Functional Ultrasmall Organic-Silica Hybrid Nanoparticles. Chem Mater. 2017; 29:6840-6855.

37. Miao Y, Benwell K, Quinn TP. 99mtc- and 111in-Labeled Alpha-Melanocyte-Stimulating Hormone Peptides as Imaging Probes for Primary and Pulmonary Metastatic Melanoma Detection. J Nucl Med. 2007; 48:73-80. [PubMed: 17204701]

38. Ow H, Larson DR, Srivastava M, Baird BA, Webb WW, Wiesner U. Bright and Stable Core-Shell Fluorescent Silica Nanoparticles. Nano Lett. 2005; 5:113-117. [PubMed: 15792423]

39. Burns A, Ow H, Wiesner U. Fluorescent Core-Shell Silica Nanoparticles: Towards "Lab on a Particle" Architectures for Nanobiotechnology. Chem Soc Rev. 2006; 35:1028-1042. [PubMed: 17057833]

40. Ma K, Sai H, Wiesner U. Ultrasmall Sub-10 Nm near-Infrared Fluorescent Mesoporous Silica Nanoparticles. J Am Chem Soc. 2012; 134:13180-13183. [PubMed: 22830608]

41. Ma K, Werner-Zwanziger U, Zwanziger J, Wiesner U. Controlling Growth of Ultrasmall Sub-10 Nm Fluorescent Mesoporous Silica Nanoparticles. Chem Mater. 2013; 25:677-691.

42. Yang P, Gai S, Lin J. Functionalized Mesoporous Silica Materials for Controlled Drug Delivery. Chem Soc Rev. 2012; 41:3679-3698. [PubMed: 22441299]

43. Larson DR, Ow H, Vishwasrao HD, Heikal AA, Wiesner U, Webb WW. Silica Nanoparticle Architecture Determines Radiative Properties of Encapsulated Fluorophores. Chem Mater. 2008; 20:2677-2684.

44. Herz E, Burns A, Bonner D, Wiesner U. Large Stokes-Shift Fluorescent Silica Nanoparticles with Enhanced Emission over Free Dye for Single Excitation Multiplexing. Macromol Rap Commun. 2009; 30:1907-1910.

45. Herz E, Ow H, Bonner D, Burns A, Wiesner U. Dye Structure-Optical Property Correlations in near-Infrared Fluorescent Core-Shell Silica Nanoparticles. J Mater Chem. 2009; 19:6341-6347.

46. Rosenkranz AA, Slastnikova TA, Durymanov MO, Sobolev AS. Malignant Melanoma and Melanocortin 1 Receptor. Biochemistry (Mosc). 2013; 78:1228-1237. [PubMed: 24460937]

47. Yang J, Guo H, Miao Y. Technetium-99m-Labeled Arg-Gly-Asp-Conjugated Alpha-Melanocyte Stimulating Hormone Hybrid Peptides for Human Melanoma Imaging. Nucl Med Biol. 2010; 37:873-883. [PubMed: 21055617]

48. Wong W, Minchin RF. Binding and Internalization of the Melanocyte Stimulating Hormone Receptor Ligand [Nle4, D-Phe7] Alpha-Msh in B16 Melanoma Cells. Int J Biochem Cell Biol. 1996; 28:1223-1232. [PubMed: 9022281] 
49. Chithrani BD, Chan WC. Elucidating the Mechanism of Cellular Uptake and Removal of ProteinCoated Gold Nanoparticles of Different Sizes and Shapes. Nano Lett. 2007; 7:1542-1550. [PubMed: 17465586]

50. Kam NW, Liu Z, Dai H. Carbon Nanotubes as Intracellular Transporters for Proteins and DNA: An Investigation of the Uptake Mechanism and Pathway. Angew Chem Int Ed Engl. 2006; 45:577581. [PubMed: 16345107]

51. Benezra M, Phillips E, Overholtzer M, Zanzonico PB, Tuominen E, Wiesner U, Bradbury MS. Ultrasmall Integrin-Targeted Silica Nanoparticles Modulate Signaling Events and Cellular Processes in a Concentration-Dependent Manner. Small. 2015; 11:1721-1732. [PubMed: 25471698]

52. Stabin MG, Sparks RB, Crowe E. Olinda/Exm: The Second-Generation Personal Computer Software for Internal Dose Assessment in Nuclear Medicine. J Nucl Med. 2005; 46:1023-1027. [PubMed: 15937315]

53. Lee FT, Scott AM. Immuno-Pet for Tumor Targeting. J Nucl Med. 2003; 44:1282-1283. [PubMed: 12902419]

54. Abou DS, Ku T, Smith-Jones PM. In Vivo Biodistribution and Accumulation of 89zr in Mice. Nucl Med Biol. 2011; 38:675-681. [PubMed: 21718943]

55. Wei L, Zhang X, Gallazzi F, Miao Y, Jin X, Brechbiel MW, Xu H, Clifford T, Welch MJ, Lewis JS, Quinn TP. Melanoma Imaging Using (111)in-, (86)Y- and (68)Ga-Labeled Chx-a" Re(Arg11)Ccmsh. Nucl Med Biol. 2009; 36:345-354. [PubMed: 19423001]

56. Holland JP, Sheh Y, Lewis JS. Standardized Methods for the Production of High Specific-Activity Zirconium-89. Nucl Med Biol. 2009; 36:729-739. [PubMed: 19720285]

57. Chen F, Ma K, Zhang L, Madajewski B, Zanzonico P, Sequeira S, Gonen M, Wiesner U, Bradbury MS. Target-or-Clear Zirconium-89 Labeled Silica Nanoparticles for Enhanced Cancer-Directed Uptake in Melanoma: A Comparison of Radiolabeling Strategies. Chem Mater. 2017; 29:82698281. [PubMed: 29123332] 
a)
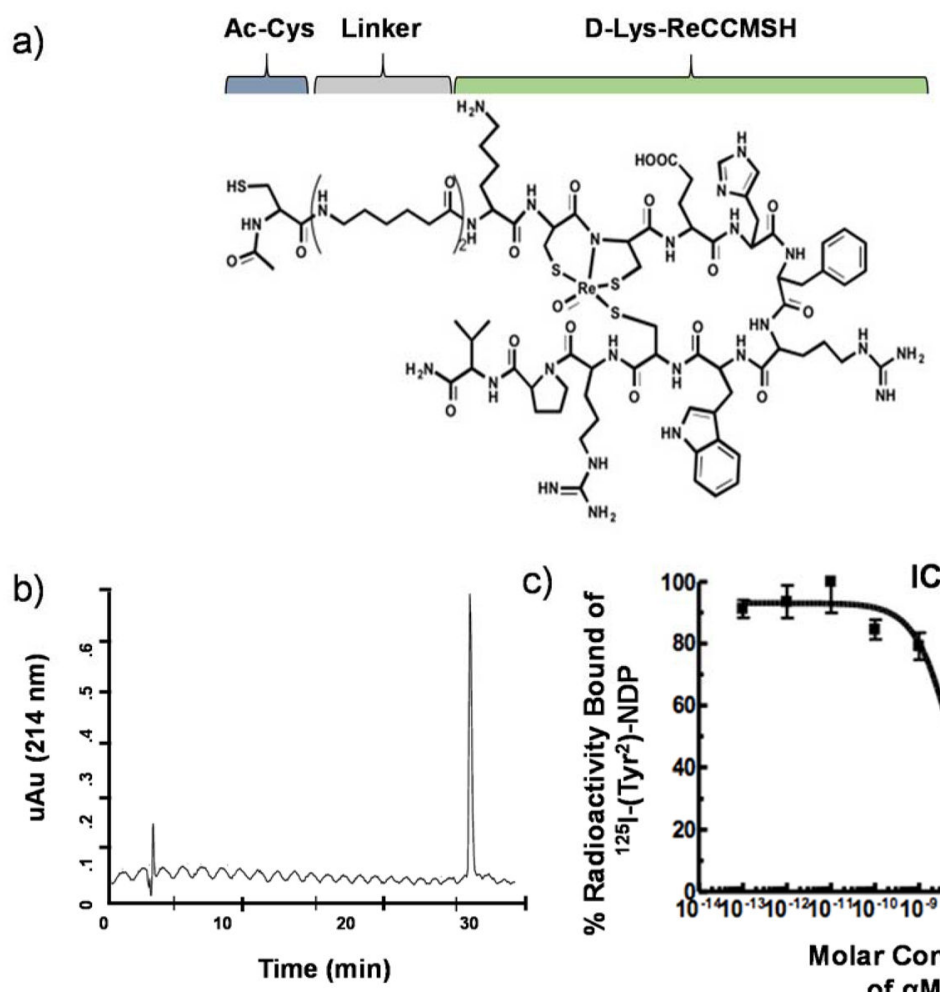

c)

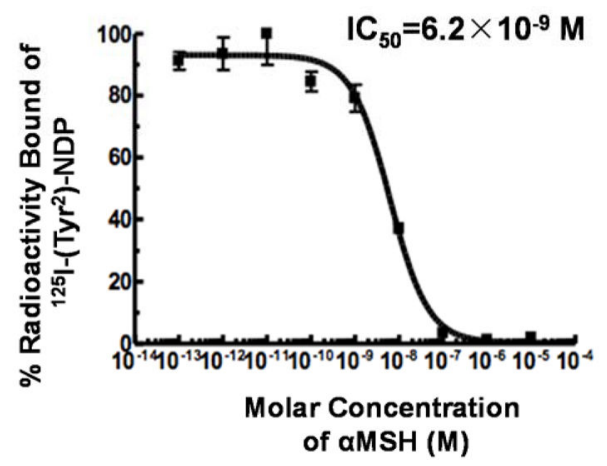

Figure 1. Characterization of the lead aMSH targeting peptide III

(a) Chemical structure of the aMSH peptide. (b) HPLC trace of the purified aMSH peptide sequence. (c) $\mathrm{IC}_{50}$ determination of the MC1-R targeting D-Lys-ReCCMSH peptide. 

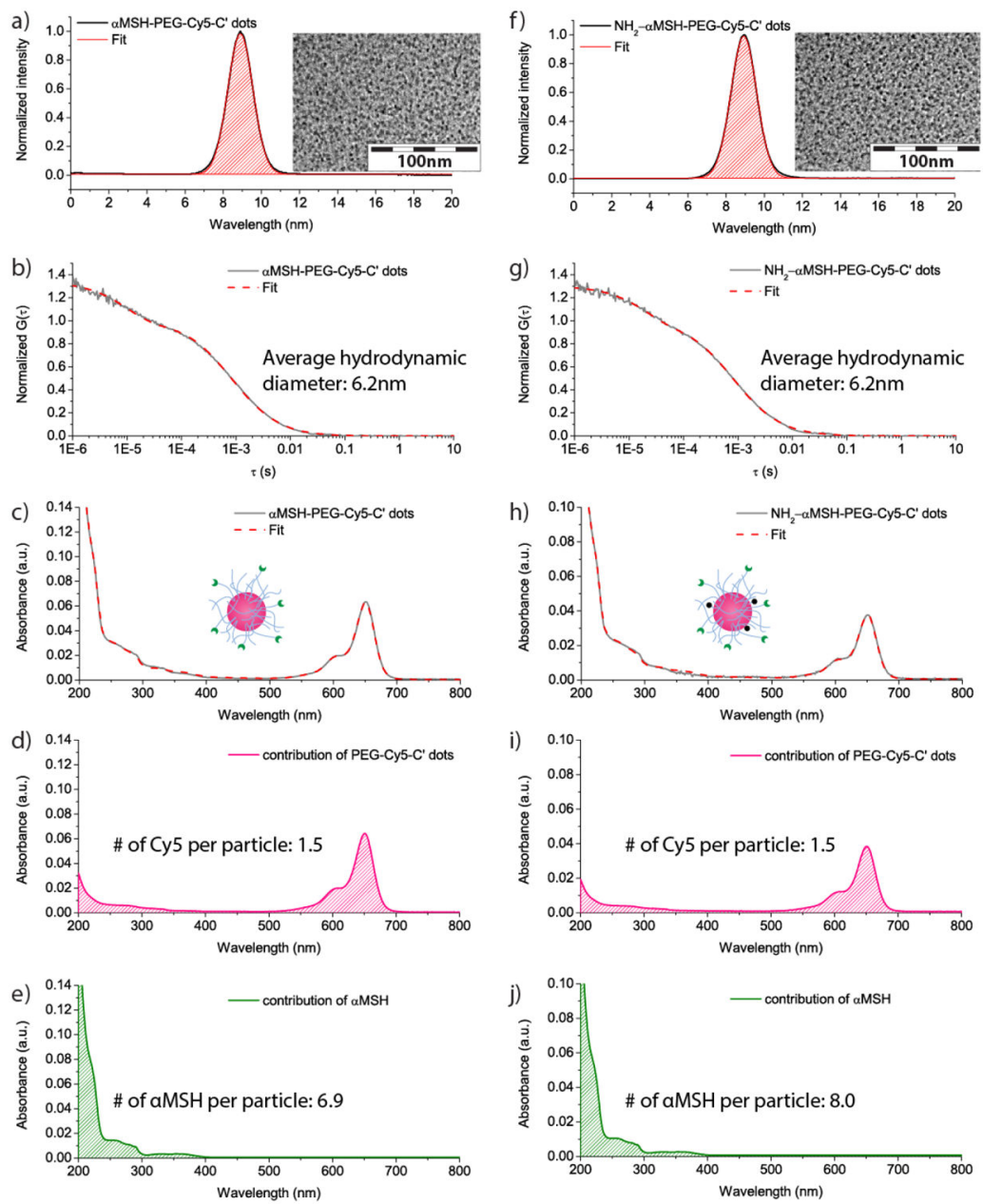

Figure 2. Characterization of aMSH-PEG-Cy5-C' ${ }^{\prime}$ dots and $\mathrm{NH}_{2}$-aMSH-PEG-Cy5-C ${ }^{\prime}$ dots (a and b) GPC elugram (a) and FCS correlation curve with fit (b) of aMSH-PEG-Cy5-C' dots. A TEM image of aMSH-PEG-Cy5-C' dots is shown in the inset of (a). (c to e) Deconvolution of the UV-Vis spectrum (c) of aMSH-PEG-Cy5- $\mathrm{C}^{\prime}$ dots into contributions from PEG-Cy5-C' dots (d) and aMSH (e). (f and g) GPC elugram (f) and FCS correlation curve with fit (g) of $\mathrm{NH}_{2}$-aMSH-PEG-Cy5-C' dots. A TEM image of $\mathrm{NH}_{2}$-aMSH-PEGCy5- $\mathrm{C}^{\prime}$ dots is shown in the inset of ( $\mathrm{f}$ ). (h to $\mathrm{j}$ ) Deconvolution of the UV-Vis spectrum (h) of NH2-aMSH-PEG-Cy5-C' dots into contributions from PEG-Cy5-C' dots (i) and aMSH (j). 
a)

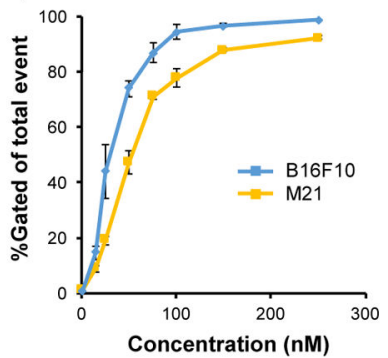

d)

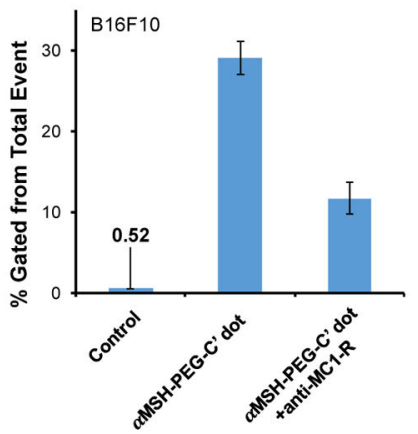

b)

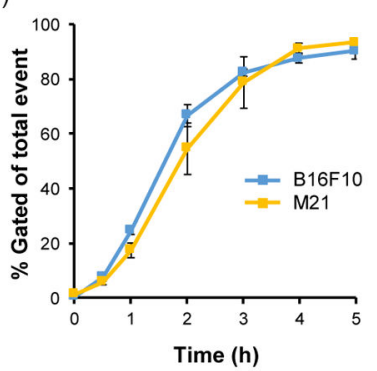

e)

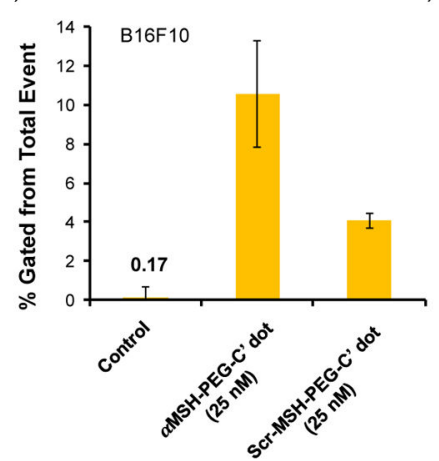

c)

f)
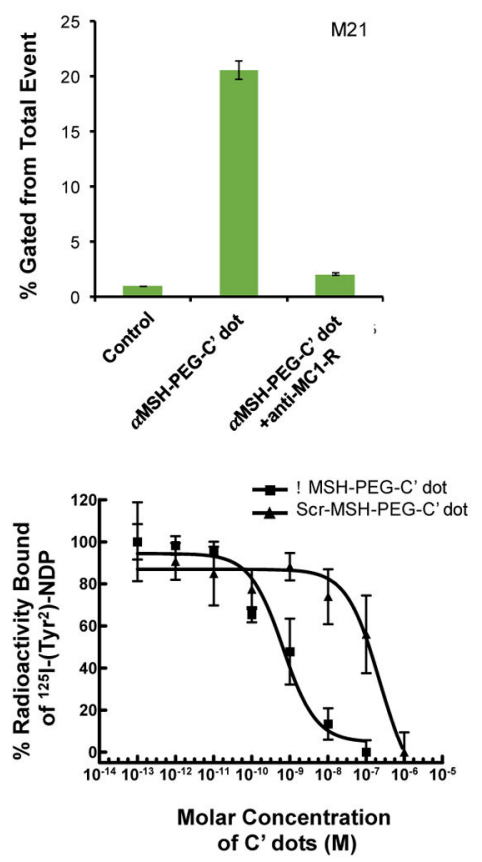

Figure 3. In vitro targeted uptake of aMSH-PEG-Cy5-C' dots and competitive binding studies in MC1-R-expressing melanoma cell lines

Cellular binding/uptake of M21 and B16F10 cells as a function of particle concentration (a) and incubation time (b) by flow cytometry. Percentage of the total events detected is displayed. Each data point represents mean \pm s.d. of three replicates. (c-e) Specific binding of aMSH-PEG-Cy5-C' dots. (c, d) MC1-R blocking of M21 and B16F10 cells, respectively, using flow cytometry and anti-MC1-R antibody prior to the addition of targeted $\mathrm{C}^{\prime}$ dots. (e) Tumor-directed binding of aMSH-PEG-Cy5-C' dots in B16F10 cells with one targeted particle concentration ( $25 \mathrm{nM}$ ) versus scrambled peptide-bound particle control, assayed by flow cytometry. Each bar represents mean \pm s.d. of three replicates. (f) Competitive MC1-R binding studies of aMSH-PEG-C'dots and Scr-aMSH-PEG-Cy5-C' dots over a concentration range of $10^{-13}$ to $10^{-5} \mathrm{~mol} / \mathrm{L}$ with ${ }^{125} \mathrm{I}-\mathrm{NDP}$ in B16/F10 melanoma cells. Each data point reflects mean \pm s.d. of three replicates. 
a)

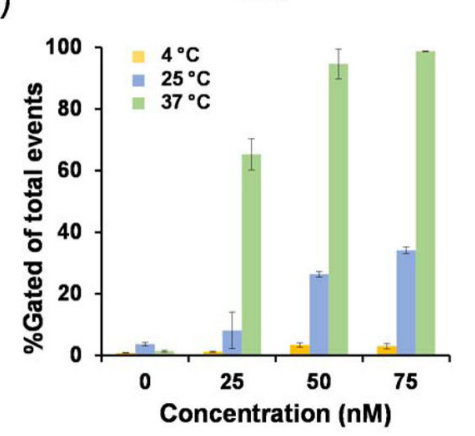

b)

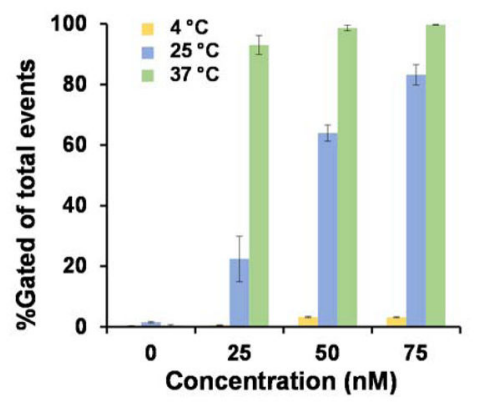

c)

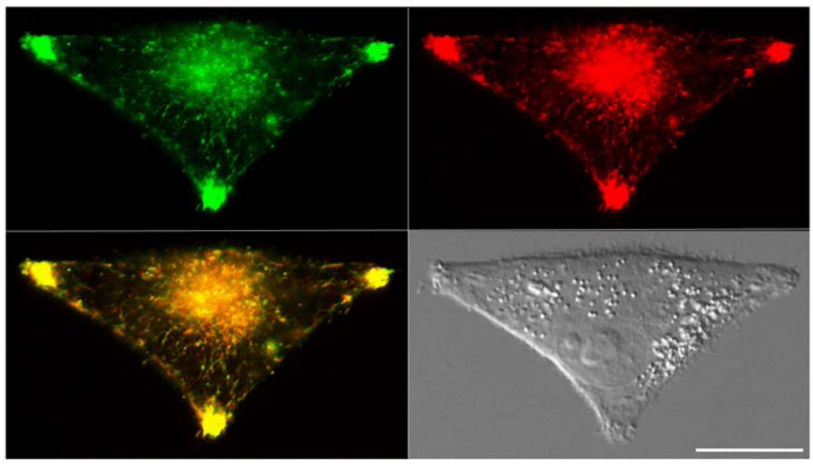

Figure 4. Temperature-dependent uptake of aMSH-PEG-Cy5-C ${ }^{\prime}$ dots and cellular internalization

Specific binding and uptake of aMSH-PEG-C dots in M21(a) and B16F10 (b) cells as a function of temperature using $25 \mathrm{nM}$ particle concentrations and flow cytometry. Each data point represents the mean \pm s.d. of 3 replicates. (c) aMSH-PEG-C ${ }^{\prime} \operatorname{dot}(1 \mu \mathrm{M}$, red) colocalization with a lysosomal marker (LAMP1-GFP) after 4h incubation using M21 cells. Colocalized vesicles (yellow); Scale bar: $10 \mu \mathrm{m}$. 
a)

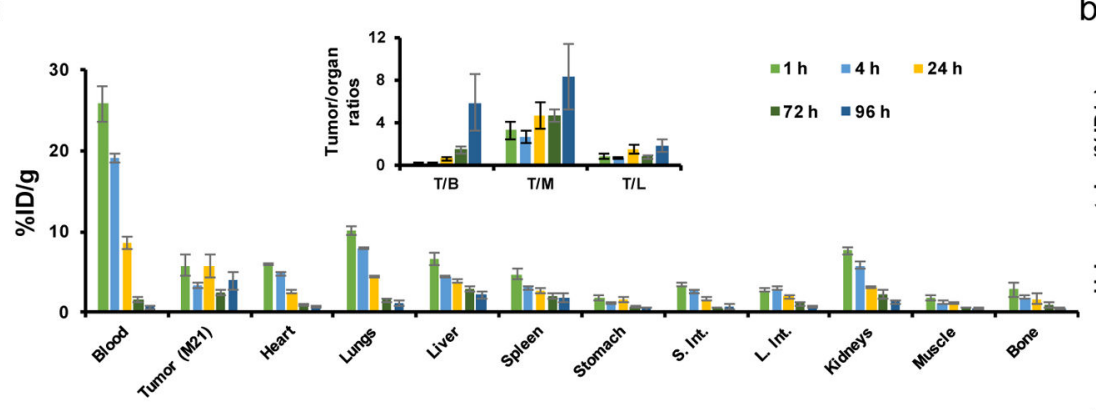

c)

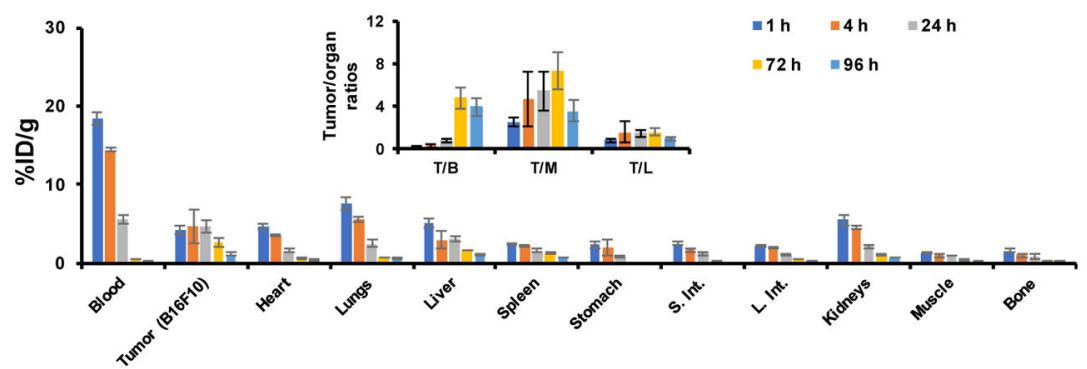

b)

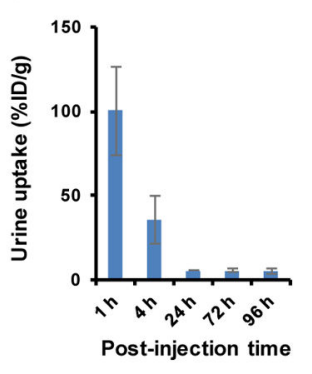

d)

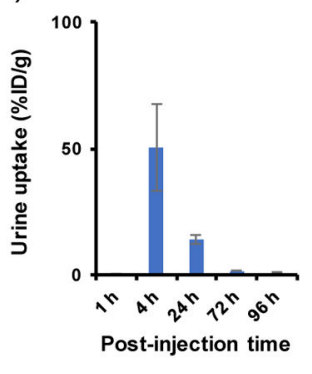

Figure 5. Biodistribution of ${ }^{125}$ I-aMSH-PEG-Cy5-C ${ }^{\prime}$ dots

in (a) M21 and (c) B16F10 tumor-bearing mice $(\mathrm{n}=3)$ at 1, 4, 24, 72 and $96 \mathrm{~h}$ post-injection. Insets shows \%ID/g values as ratios for tumor to organ (T/B: tumor to blood; T/M: tumor to muscle; T/L: tumor to liver). (b) and (d) are the corresponding activities of ${ }^{125} \mathrm{I}-\mathrm{aMSH}$ PEG-Cy5-C' dots in urinary specimens. 
a)

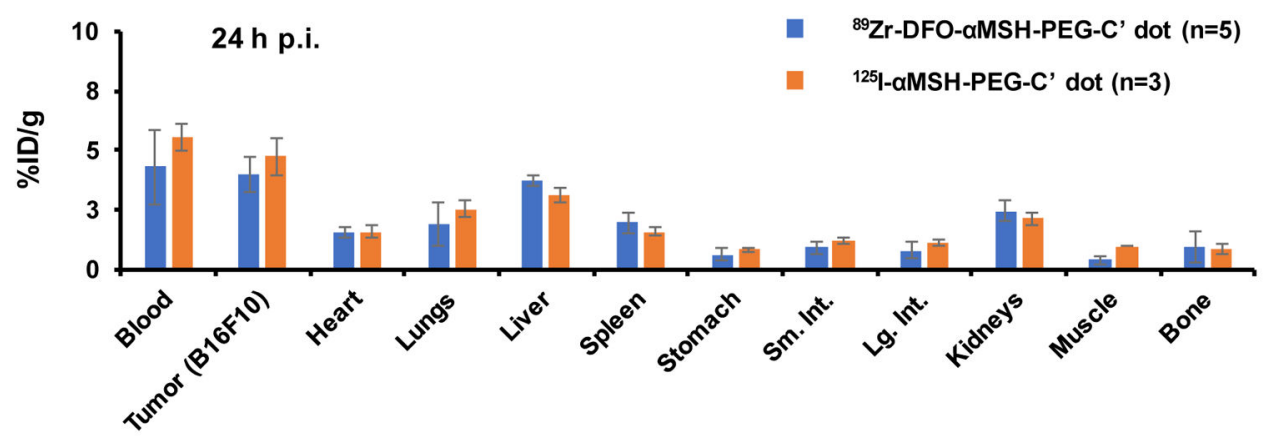

b)

H\&E

Optical (Cy5)

Autoradiography
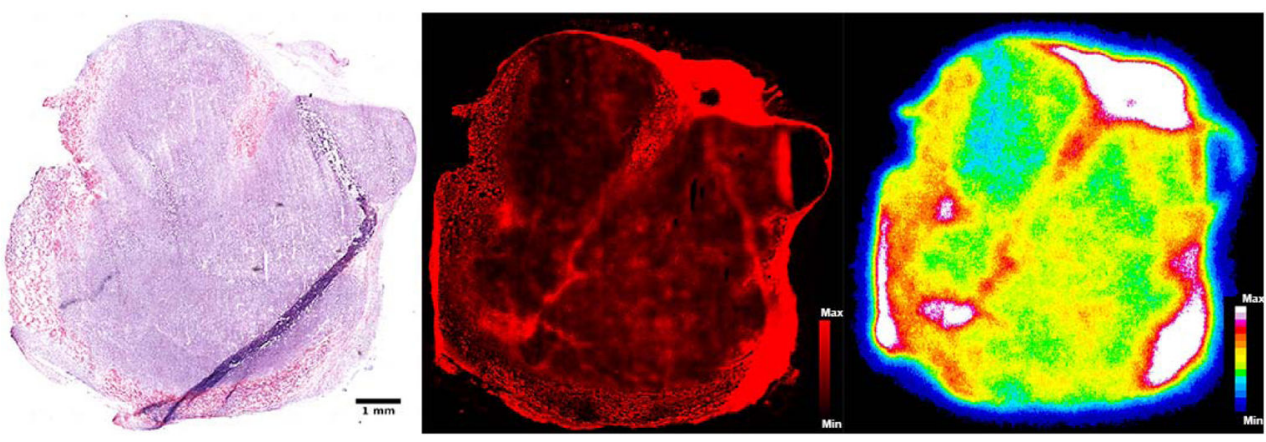

Figure 6. Biodistribution and ex vivo histological studies

(a) Comparison of the biodistribution profiles in $\mathrm{B} 16 \mathrm{~F} 10$ tumor-bearing mice $24 \mathrm{~h} \mathrm{p.i.} \mathrm{of}$ ${ }^{89} \mathrm{Zr}$-DFO-aMSH-PEG-C ${ }^{\prime} \operatorname{dot}(\mathrm{n}=5)$ or ${ }^{125} \mathrm{I}$-aMSH-PEG-C' ${ }^{\prime} \operatorname{dot}(\mathrm{n}=3)$; (b) H\&E staining (left, scale bar: $1 \mathrm{~mm}$ ), Cy5-fluorescence microscopy (middle), and autoradiography (right) images of tumor tissue specimen harvested from B16F10 tumor-bearing mouse $24 \mathrm{~h} p . i$. of ${ }^{89} \mathrm{Zr}-\mathrm{DFO}-\mathrm{aMSH}-\mathrm{PEG}-\mathrm{Cy} 5-\mathrm{C}^{\prime}$ dots. 
a)

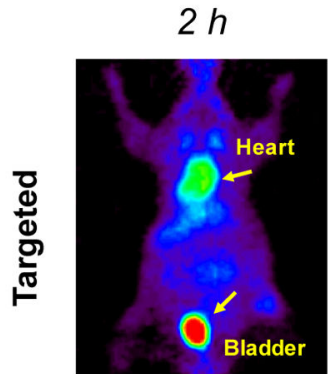

b)

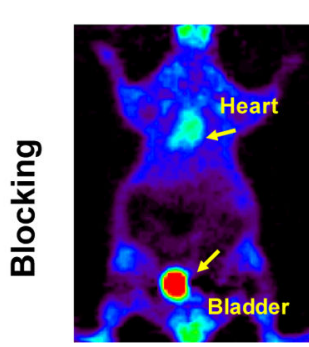

$20 \% \mathrm{ID} / \mathrm{g}$
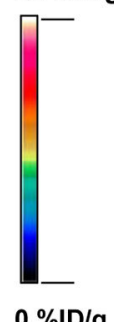

Figure 7. Serial PET imaging of ${ }^{89} \mathrm{Zr}$-DFO-aMSH-PEG-Cy5-C ${ }^{\prime}$ dots at 2, 24, 48 and $72 \mathrm{~h}$ post-injection of the MC1-R-targeting particle tracer into two cohorts of B16F10 xenografted mice: (a) targeted group and (b) NDP blocking group. For the blocking study, each mouse was co-injected with ${ }^{89} \mathrm{Zr}-\mathrm{DFO}-\mathrm{aMSH}-\mathrm{PEG}-\mathrm{Cy} 5-\mathrm{C}^{\prime}$ dots and $200 \mu \mathrm{g}$ of NDP. $\mathrm{N}=3$ for each group. 
Targeted

a)

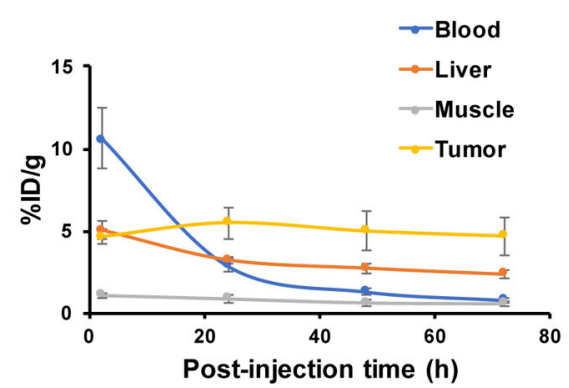

c)

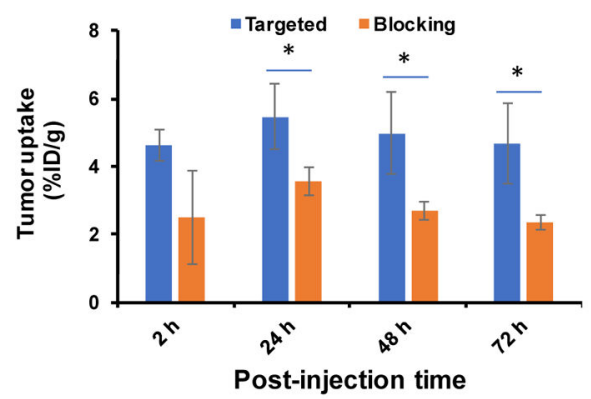

e)

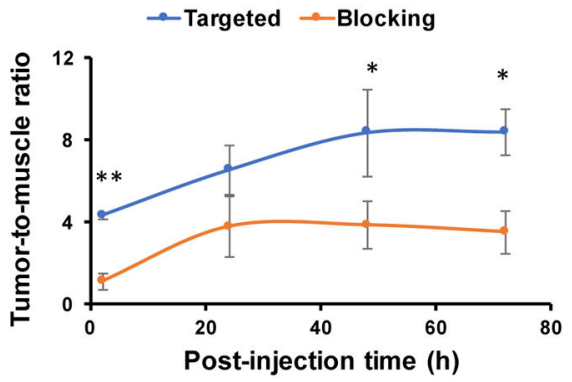

Blocking

b)

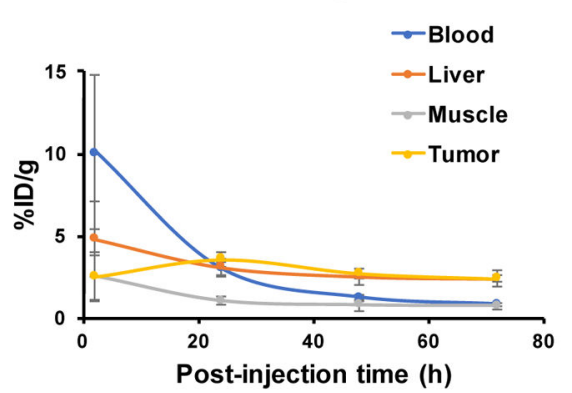

d)

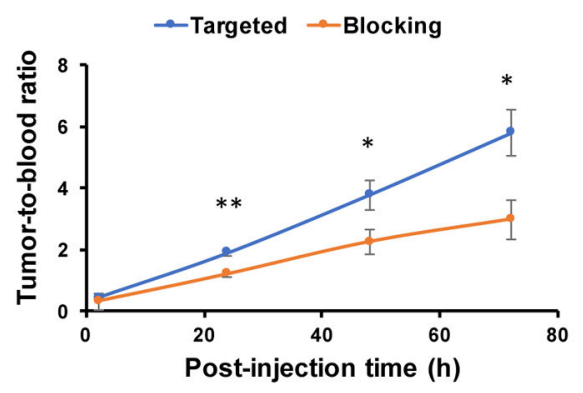

f)

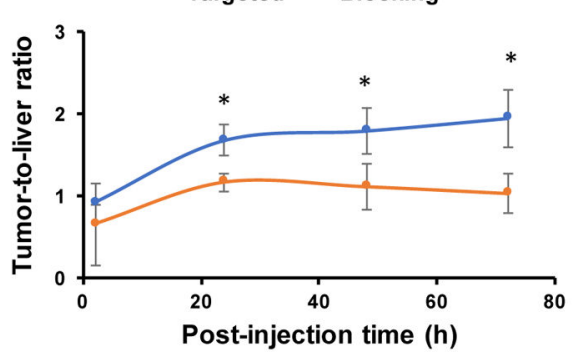

Figure 8. Quantitative region-of-interest studies in B16F10 xenografted mice

Time activity curves of (a) targeted group ( $n=3)$, and (b) blocking group $(n=3)$. (c) Tumor uptake comparison between targeted and blocking groups $(n=3)$. (d-f) Tumor-to-blood, tumor-to-muscle and tumor-to-liver ratios at 2, 24, 48 and $72 \mathrm{~h}$ post-injection. For blocking study, the mice were co-injected with ${ }^{89} \mathrm{Zr}-\mathrm{DFO}-\mathrm{aMSH}-\mathrm{PEG}-\mathrm{Cy} 5-\mathrm{C}^{\prime}$ dots and $200 \mu \mathrm{g}$ of NDP. $* \mathrm{P}<0.05, * * \mathrm{P}<0.005$. 


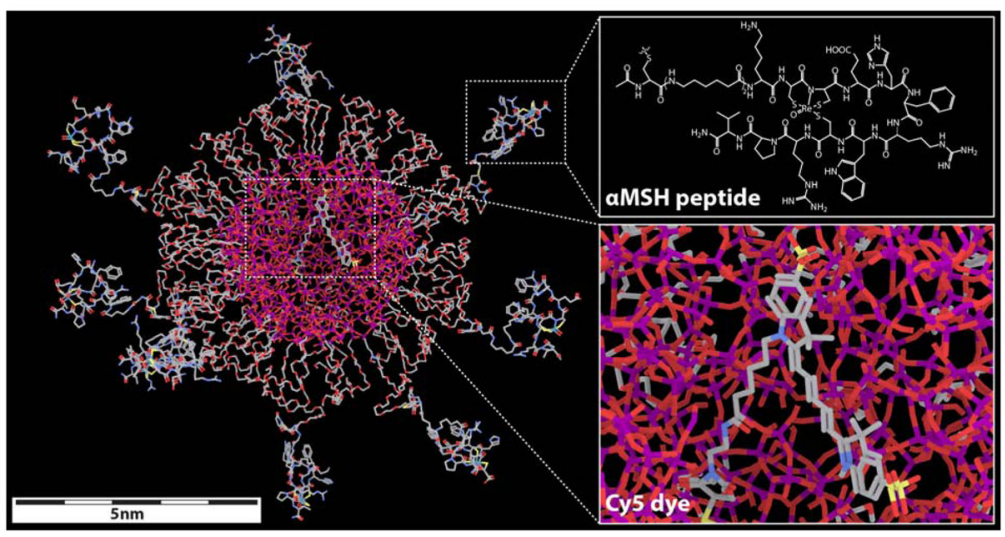

Scheme 1. Molecular rendering of aMSH-PEG-Cy5-C' dots

Left side shows rendering of the entire dot, with inserts on the right depicting aMSH chemical structure (top) and encapsulated Cy5 dye (bottom). Silicon, oxygen, carbon, nitrogen, sulfur and rhenium atoms are colored in purple, red, gray, blue, yellow and light green, respectively. Hydrogen atoms are not displayed in the model for better visualization. 
Table 1

\section{Radiodosimetry of ${ }^{125} \mathrm{I}$-aMSH-PEG-Cy5-C ${ }^{\prime}$ dots}

in a 70-kg standard human based on the pharmacokinetics in M21 and B16F10 tumor-bearing mice and the OLINDA dosimetry program.

\begin{tabular}{lcc}
\hline Tissue & \multicolumn{2}{c}{ Absorbed Dose (mGy/MBq) } \\
& Based on M21 biodistribution data & Based on B16F10 biodistribution data \\
Adrenals & 0.204 & 0.206 \\
Brain & 0.140 & 0.168 \\
Breasts & 0.129 & 0.147 \\
Gallbladder Wall & 0.223 & 0.224 \\
Lower Large Intestine Wall & 0.276 & 0.263 \\
Small Intestine & 0.243 & 0.248 \\
Stomach Wall & 0.270 & 0.247 \\
Upper Large Intestine Wall & 0.249 & 0.240 \\
Heart Wall & 0.206 & 0.206 \\
Kidneys & 0.324 & 0.242 \\
Liver & 0.335 & 0.251 \\
Lungs & 0.249 & 0.192 \\
Muscle & 0.179 & 0.169 \\
Ovaries & 0.204 & 0.226 \\
Pancreas & 0.215 & 0.220 \\
Red Marrow & 0.212 & 0.193 \\
Bone & 0.354 & 0.308 \\
Skin & 0.115 & 0.128 \\
Spleen & 0.262 & 0.195 \\
Testes & 0.152 & 0.178 \\
Thymus & 0.164 & 0.178 \\
Thyroid & 0.161 & 0.179 \\
Urinary Bladder Wall & 0.281 & 0.359 \\
Uterus & 0.206 & 0.243 \\
Total Body & $\mathbf{0 . 1 9 2}$ & $\mathbf{0 . 1 9 4}$ \\
Effective Dose & $\mathbf{0 . 2 2 2}$ & $\mathbf{0 . 2 2 3}$ \\
\hline
\end{tabular}

